\title{
UNITED STATES \\ PRIVATE TOBACCO \\ PROPRIETARY STAMPS
}
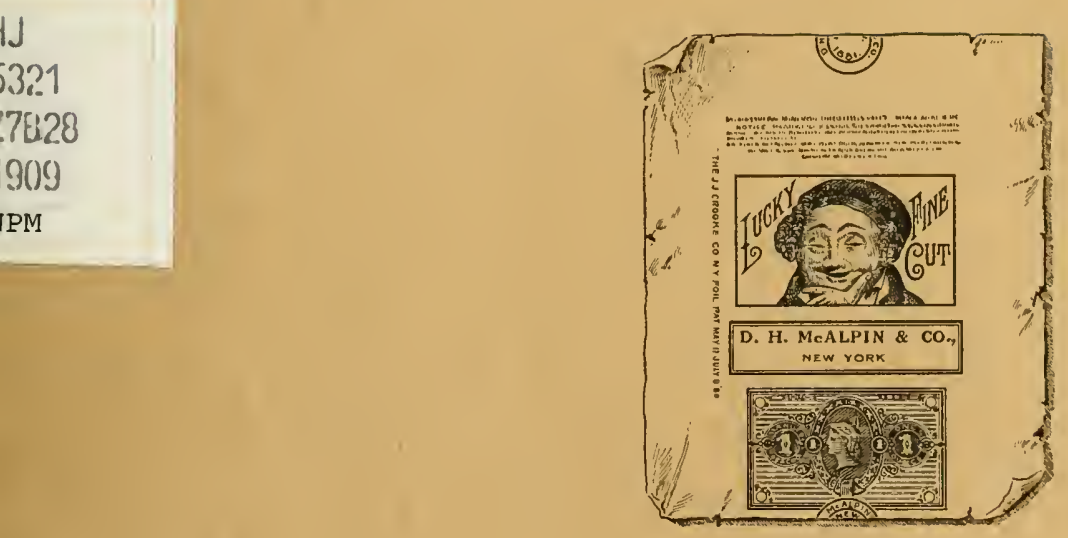

J. DELANO BARTLETT JOHN W. PREVOST 


\section{UNITED STATES PRIVATE TOBACCO PROPRIETARY STAMPS}

PRINTED ON TIN FOIL

B)

THE J. J. CROOKE CO. AND

THE COULEY FOIL CO.
PRINTED ON PAPER

$\mathrm{B} \mathrm{C}^{\circ}$

THE CONTINENTAL BANK NOTE CO. AND

THE GRAPHIC CO.

INCLUDING

ILI KNOWN ESA I Y IND I LISTOF FOILS TAKEN FPOM THE J. I. CROOKE CO. RECORDS BY ALFRED A. POST. 


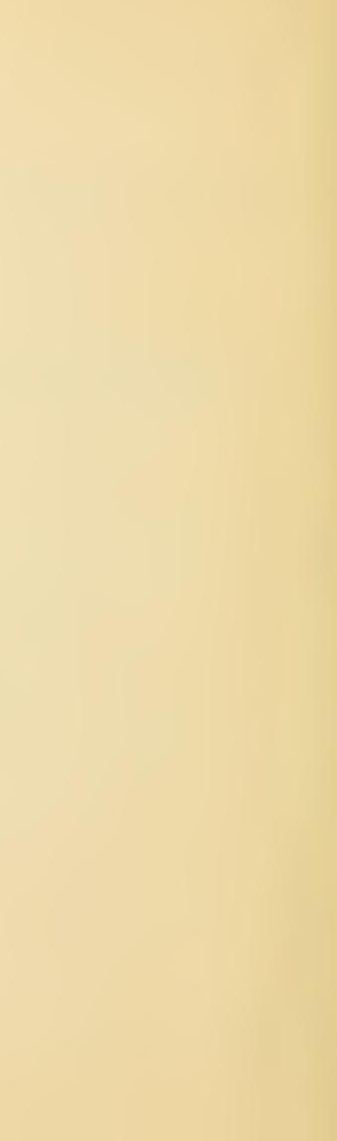




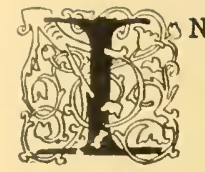

issuing this list of Private Tobaceo Proprietary Stamps, we leel that our efitorts will be alphereiaterl hy all colleetors who are interested in United States Revenue Stamps, as we know of no list of these stamps published sinee August, 188\%. We have used the utmost eare in our work and have isted only those, which we know to have hern in the possession of some collector. "l'he same call be sald of the esisalys.

The list contained on the last eight pages was taken from the records of the .J. J. Crooke ('om. billy [Foil l'rinters | by dlfred A. Post as work done by them, but there is nothing to show that these were stamp-

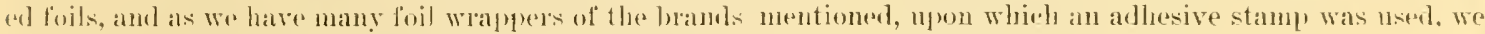
are inclinerl to believe that they were all of that class.

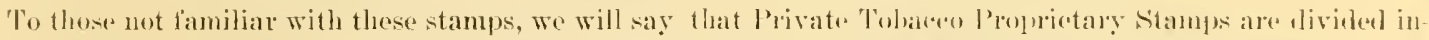
to two classes. First: With stamp and brand printed on tin foil. These were issued in 1868 and are still in use. Seronel: With stamp and brand printed on paper. These were also first isoned in 1868 and weres prined loy the continneltal Bank Note Company. This issue was in use for a very brief period. The next issue appeared in $1 s 78$, and was printed by the Graphic Company. This was discontiuned in 1851.

There is one thing which should be noted calrefully in following this list. ( )n all foils the proprietor's nane is the one inscribed in the circle containing the date, witlent any jegard to the proprietor's name given whewlere on the foil, as in many cases, brand owners sold ont and the new proprictor retained the old brand and name, but lis namor was always inscribed in the date circle.

Wra are inclebted to Mr. II. F. Deats for the privilege of examining one of his books of tin foils.

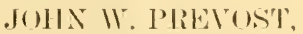

J. DHLANG BAR'TIET'T'. 

I. $100 \mathrm{NCH}$

\begin{tabular}{|c|c|c|c|c|c|c|c|}
\hline Nor. & I'roprietor & IIrнü & & IHeiriei & folorr & Kind of Fons & ve. \\
\hline 1 & Allen, Reynolds \& Co. & Indian Weed & & 4th N.J. & Blue & Bright & 1 \\
\hline 2 & Alken, Tibly \& C'o. & Jndian Belle & & jth N.J. & 131u世" & I3right & 2 \\
\hline 3 & Anos, D. B. & Crystal & & Ioth N.Y. & Blue & Bright & 3 \\
\hline 4 & Bagley, John J. \& C'o. & Mayflower & - & 1st Mich. & Blue & Bright & 4 \\
\hline 5 & Douglass, I. S. & Virginia leaf & & $3 \mathrm{~d} N . Y$ & Blut & Bright & 5 \\
\hline 6 & Gillender, Arthur \& Co. & Fragrant & (Bhu, & $32 \mathrm{~d} N . \mathrm{Y}$ & Blue & Bright & b \\
\hline 7 & Gillender, Arthur \& Co. & Fragrant & (Gold) & $32 \mathrm{~d}$ N.Y. & Blue & Bright & ; \\
\hline s & Goodwin, Wm. H. \& Co. & Plantation & & $32 \mathrm{~d}$ N.Y. & Blue & Bright & $s$ \\
\hline 9 & Greer, Alex \& Son & Cavendish & & 1 th N.Y. & Blue & Bright & 9 \\
\hline 10 & Hoyt, Thos. \& J. F. Flagg & Heart's Delight & (Brown) & $32 \mathrm{~d}$ N.Y. & Blue & Bright & 10 \\
\hline 11 & Hoyt, Thos. \& J. F. Flagg & National & & $32 d N . Y$ & Blue & Bright & 11 \\
\hline 12 & Langenbach, C. & Planet & & 5th X.Y. & Blue & Bright & 12 \\
\hline 13 & Lichtenberg, G. B. & Violet & & 1 st Mich. & Blue & Bright & 13 \\
\hline 14 & Lilienthal, C. H. & Neptune & & $2 \mathrm{dN.Y.}$ & Blue & Bright & 14 \\
\hline 15 & Lilienthal, C. H. & Septune & & th N.Y. & Blue & Bright & 15 \\
\hline 16 & Lorillard, P. \& Co. & Eureka & & $2 \mathrm{dN.Y}$ & Blue & Bright & 16 \\
\hline 17 & Lorillard, P. \& Co. & Eureka & & tth N.Y. & Blue & Bright & 17 \\
\hline 18 & Lorillard, P. \& Co. & Olive Branch & & $2 \mathrm{~d} N . Y$ & Blue & Bright & in \\
\hline 19 & Lorillard, P. \& Co. & Olive Branch & & th N.Y. & Blue & Bright & 19 \\
\hline 20 & McAlpin, D. H. \& Co. & Jehn Cornish Virgin Leaf & (7.) Buwery) & sth X.Y. & Blue & Bright & 20 \\
\hline 21 & MeAlpin, D. H. \& Co. & John Cornish Virgin Leat & (75etcolve.1n) & ith N.Y. & Blue & Bright & 21 \\
\hline 22 & MeAlpin, D. H. \& Co. & John Cornish Virgin Leaf & (1tisetc.uve.D) & Tth N.Y. & Blue & Bright & 22 \\
\hline 23 & MeAlpin, D. H. \& Co. & Jehn Cornish Virgin Leaf & (1fti erc.A (ve. D) & Id X.Y. & Blue & Bright & 23 \\
\hline 24 & IlcAlpin, D. H. \& Co. & Navy & ete. Are. Di & 7th N.Y. & Blıe & Bright & 24 \\
\hline 25 & IICAlpin, D. H. \& Co. & Nary & ete. Ave. D) & 7th N.Y. & Blue & Bright & 25 \\
\hline
\end{tabular}


1868 ISSUE. "Class 32 cts." (Continued).

FOILS.

1/2 OUNCE.

\begin{tabular}{|c|c|c|c|c|c|c|c|}
\hline No. & Propurietor & Brand & & Distriet & Color & IFind of Foll & No. \\
\hline 26 & McAlpin, 1. H. \& Co. & Navy & $(1+b$ etc. Ave. D) & $3 \mathrm{~d} N \mathrm{X}$. & Blue & Bright & 26 \\
\hline 27 & Miller, Mrs. G. B. & Girape & & 7 th N.Y. & Blue & Bright & 27 \\
\hline 28 & Nevin \& Mills & Banner & & 1st Mich. & Blue & Bright & 28 \\
\hline 29 & Platts, Gaskin \& Patterson & Metamora & & 26 th N.Y. & Blue & Bright & 29 \\
\hline 30 & ScheIder, Jos. & Dominion & & 5th N.Y. & Blue & Bright & 30 \\
\hline 31 & Scheider, Jos. \& Co. & Dominion & & sth N.Y. & Blue & Bright & $3 I$ \\
\hline 32 & Watts, H. H. & Virsinia Leaf & & 7 th N.Y. & Blue & Bright & 32 \\
\hline 33 & Weed \& Co. & Telocipede & & ith N.Y. & Blue & BrIght & 33 \\
\hline
\end{tabular}


$10 \mathrm{CN}$.

\begin{tabular}{|c|c|c|c|c|c|c|c|}
\hline No. & Propriteior & แruna & & Dinerict & (w)or & latul of toit & 16. \\
\hline 1 & Allen, Reynolds \& Co. & liagnolia & & :t: X.J. & $1 ; 011$ & Bright & I \\
\hline 2 & Iagley, John J. \& Co. & Wayflower & & l: $\mathrm{t}: \mathrm{j} c \mathrm{~h}$ & $\therefore e x:$ & Bright & 2 \\
\hline 3 & Barher, K. C. & Anericau Eagle & & I t lich.h. & $\therefore 011$ & Bright & 3 \\
\hline 4 & Barker, K. C. & ('ippes. & & lit Wich. & r ol.1 & Eright & 4 \\
\hline 5 & Lronson \& Messenger & Faney & (B]ue) & 10th Ohio & 6.11 & Bright & 5 \\
\hline 6 & Bronson \& Mrosenger & Granger & (Blue) & 10th Onio & Ciold & Bright & (i \\
\hline 7 & Buchner, D. & Natural Leaf & (1.eaf Itp) & ith N.Y. & $6,01.1$ & Briglit & ? \\
\hline 8 & Buchner, $\mathrm{D}$. & Natural Leaf & 'Leaf Down' & Iih N.Y. & rivld & Bright & 8 \\
\hline 9 & Cotterill, Feuner \& Co. & Velocipede & & $3+1$ Ohio & Ciolit & Bright & 9 \\
\hline 10 & Douglass, I. S. & Feerless ('hewins & & $2 \mathrm{~d} X Y$. & f.old & Bright & 10 \\
\hline 11 & Douglass, I. S. & Golden & (Factory Yo. 21) & $2 \mathrm{~d}$ N.Y. & Col. & Bright & 11 \\
\hline 12 & Douglass, I. S. & Specie & (Fuctory No. 3) & id X.Y. & c,old & Bright & 12 \\
\hline 13 & Edmondston, Eamıs. & Golden Stat & & th X.S. & (igld & Briglit & 13 \\
\hline 14 & Edmouston, S. S. \& Bro. & Phoenix & & fth X.Y. & Ciold & Briglit & 14 \\
\hline 15 & Edmonston, S. S. \& Bro. & Phoenix & & 2d X.Y. & Colt & Bright & 15 \\
\hline 16 & Edmonston, S. S. \& Bro. & Pride of the $[$. S & & $2 \mathrm{~d} \mathrm{N.Y.}$ & Gold & Bright & 16 \\
\hline 17 & Gillender, Arthur \& Co. & Solace $\quad(\cdots T e n$ & Cents" small lefters & $32 d$ N.Y. & Gold? & Bright & 17 \\
\hline 18 & Gillender, Arthur \& Co. & Solace $(T \in n$, ent & its) (Inscription in 2 lines) & $32 \mathrm{~d} \mathrm{N.Y.}$ & Gold & Bright & 18 \\
\hline 19 & Gillender, Arthur \& Co. & Solace t Ten $\cdot \operatorname{tent}$ & ts) (Inscription in 3 lines) & $32 d \mathrm{NYY}$ & Gold & Bright & 19 \\
\hline 2) & Gillender, Arthur \& Co. & Solace & without "Trn Cents") & : :บ X.Y. & Gold & Bright & 20 \\
\hline $2 !$ & Gooiwin, IV. H. \& Co. & Yellow Bank & ("Pure" large type) & $32 \mathrm{~d}$ N.Y. & Gold & Bright & 21 \\
\hline 22 & Goodwin, W. H. \& Co. & Yellow Bank & ("Pure" smail type) & $32 d$ N.Y. & Gold & Bright & 22 \\
\hline 23 & Goodwin \& Co. & Yellow Bank & ("Pure" linge type) & $3 \geq \mathrm{d} N . \mathrm{Y}$ & Cold & Dright & 23 \\
\hline 21 & Iloyt. Thos. \& .J. F. Flagg & Heart's Deligit & & $32 \mathrm{~d}$ X.Y. & rolul & $\mathrm{Bri}$ & 24 \\
\hline 271 & T.angenbach, C. & Consoler & & ith N.Y. & rinid & Briglit & 25 \\
\hline
\end{tabular}


1868 ISSUE. "Class 32 cts." (Continued).

\section{OUNCE.}

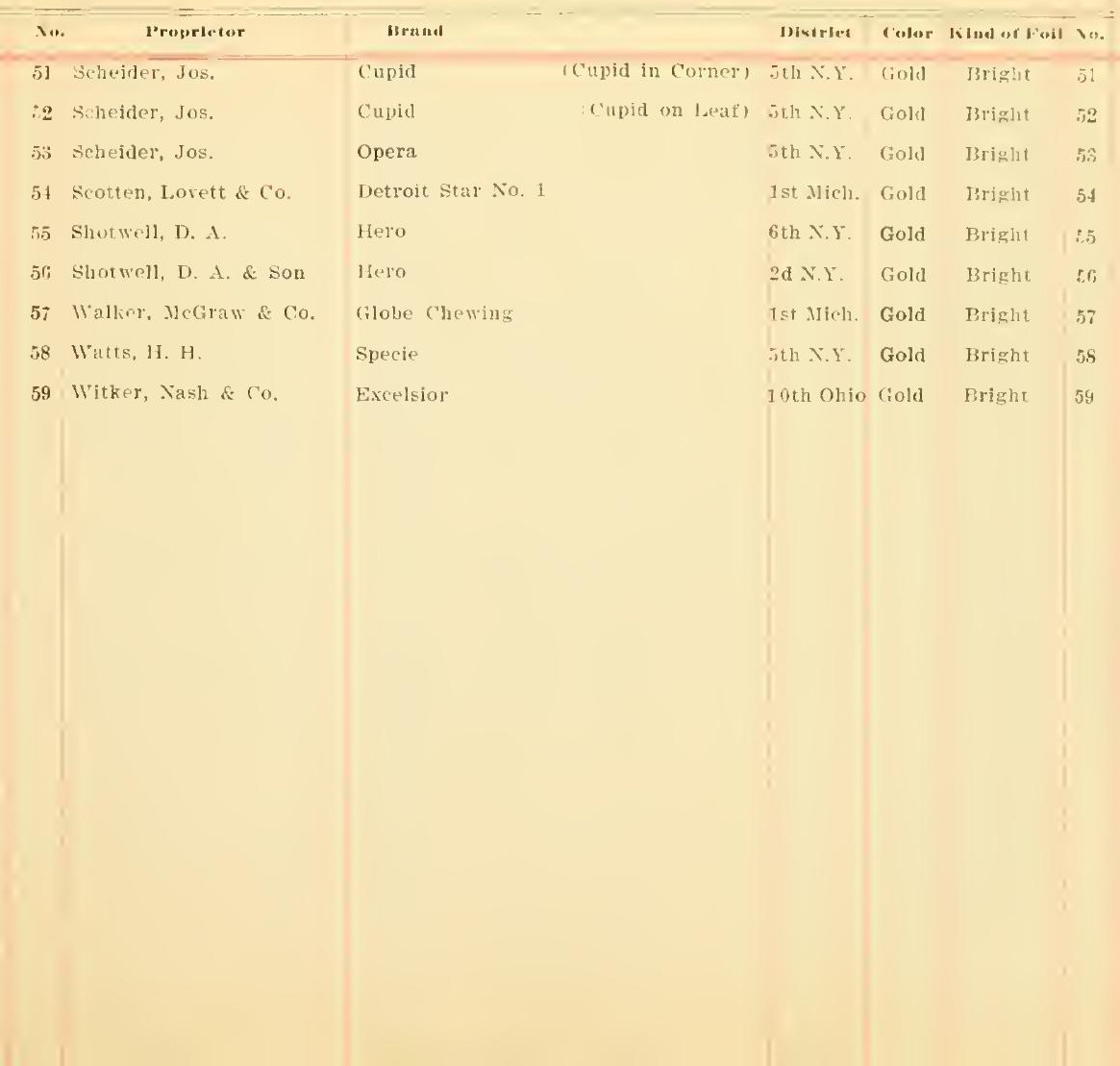


$10 \mathrm{~N} \mathrm{NCE}$.

\begin{tabular}{|c|c|c|c|c|c|c|c|c|}
\hline No. & Proprietor & Mrauı & & & Dintriet & Color & InInI of leoll & No. \\
\hline 1 & Allen \& bunning & Magnolia & & & 4th N.J. & Gold & Bright & 1 \\
\hline 2 & Anderson, John \& Co. & Solace & & (Ten Cents) & $32 \mathrm{~d} N . \mathrm{Y}$ & Gold & Bright & 2 \\
\hline 3 & Anderson, John \& Co. & Solace & without "'T & Ten (ents") & $32 \mathrm{~d}$ N.Y. & Gold & Bright & 3 \\
\hline 4 & Anderson, John \& Co. & Solace & ( For & Californial & $32 \mathrm{~d}$ N.Y. & Gold & Bright. & 4 \\
\hline 5 & Bagley, John J. \& ('o. & Gold Dust & & & Ist Mich. & Gold & Bright & 5 \\
\hline 6 & Bagley, John J. & Nayflower & & & 1st Mich. & Gold & Bright & 6 \\
\hline 7 & Bagley, John J. & Sunshine & & & Ist Mlich. & Blue & Bright & 7 \\
\hline 8 & Barker, K. C. & American Eagle & & & Ist Mich. & Gold & Bright & 8 \\
\hline 9 & Barker, K. C. & Clipper & & & 1 st Mich. & Gold & Bright & 9 \\
\hline 10 & Barker, K. C. & Eureka & & & Ist Mich. & Giold & Bright & 10 \\
\hline 11 & Barker, K. C. & Golden Luxury & & & 1st Mlich. & Gold & Bright & 11 \\
\hline 12 & Buchner, D. & Natural Leaf & & (Leaf Up) & $3 d \mathrm{~N} . \mathrm{Y}$ & Gold & Bright & 12 \\
\hline 13 & Camphell, Lane \& Co. & Golden Light & & & $5 \operatorname{ch~N.J.~}$ & Gold & Bright & 13 \\
\hline 14 & Christman, F. \& Co. & Highland Gem & & & 6th lnd. & Gold & Bright & 14 \\
\hline 15 & Cotterill, Fenner \& Co. & King Bee & & & 3.1 Ohio & Gold & Bright & 15 \\
\hline 16 & Cotterill, Fenner \& Co. & North Star & & & $3 \mathrm{~d}$ Ohio & Gold & Bright & 16 \\
\hline 17 & Cotterill, Fenner \& Co. & Velocipede & & & 3 U Ohio & Gold & Bright & 17 \\
\hline 18 & Doll \& Co. & Silver Lake & & & $5 \operatorname{th~} \mathrm{Ky}$. & Gold & Bright & 18 \\
\hline 19 & Dunning \& Co. & Magnolia & & 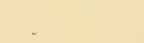 & tth N.J. & Gold & Bright & 19 \\
\hline 20 & Edmonston, Sam'l S. & Golden Quid & & & $2 \mathrm{~d} N . Y$ & Gold & Bright & 20 \\
\hline 21 & Edmonston, Sam'l S. & Golden Seal & & & $2 \mathrm{~d} \mathrm{N.Y.}$ & Gold & Bright & 21 \\
\hline 22 & Finley, Doll \& ('o. & Falls of Ohio & & & jth $\mathrm{Ky}$. & Gold & Bright & 22 \\
\hline 23 & Finley, woll \& Co. & Silver Lake & & & jth ky. & Gold & Bright & $2:$ \\
\hline 24 & Flagg, J. F. \& Co. & Seaside & & & Ist N.Y. & Gold & Bright & 24 \\
\hline 25 & Glore, J. A. P. \& Co. & Corn Cracker & & & 6th N.Y. & Gold & Bright & $2 i$ \\
\hline
\end{tabular}


1. UTNCE.

\begin{tabular}{|c|c|c|c|c|c|c|c|}
\hline No. & Proprietor & IIrand & & Disiriet & Cober & Kind of rioll & No. \\
\hline 26 & Goodwin \& Co. & ( Dark & (Weleome) & $32 \mathrm{~d}$ N.Y. & Gold & Bright & 26 \\
\hline 27 & Goodwin \& Co. & Bright WeIcome & (Light) & $32 d$ N.Y. & Gold & Bright & 27 \\
\hline 28 & Goodwin \& Co. & Plantation & & $32 d \mathrm{~N} . Y$. & Blue & Bright & $2 s$ \\
\hline 29 & Goodwin \& Co. & Solitaire & & $32 \mathrm{~d} \times \mathrm{Y}$. & Gold & Bright & 29 \\
\hline 30 & Goodwin \& Co. & Welcome & (Darli) & $32 \mathrm{~d}$ N.Y. & Gold & Bright & 30 \\
\hline 31 & Goodwin \& Co. & Yellow Bank & & $32 \mathrm{~d} N . Y$. & Gold & Bright & 31 \\
\hline 32 & Greer's. Alex. Sons & Carendish & & 14 th X.Y. & Gold & Bright & 32 \\
\hline 38 & Hoyt, Thos. \& Co. & Nabob Chewing & & $32 \mathrm{~d} \times \mathrm{Y}$. & Blue & Bright & 33 \\
\hline 34 & Kenny, Lewis & Senex & & sth N.J. & Gold & Bright & 34 \\
\hline 35 & Kimball, Wm. S. \& Co. & Idaho & & & Blue & Bright & 35 \\
\hline 36 & Leidersdorf, B. \& Co. & Empire & & 1st Wis. & Blue & Bright & 36 \\
\hline 37 & Lichtenberg, G. B. & Lightening & ( $(\mathrm{ew}$ York) & $2 \mathrm{~d}$ N.Y. & Gold & Bright & 37 \\
\hline 38 & Lichtenberg, G. B. & Morning Glory & (Detrnit) & 1 st Mich. & Gold & Bright & 38 \\
\hline 39 & Lichtenberg, G. B. & Morning Glory & (New York) & $2 \mathrm{~d} X \mathrm{Y}$. & Gold & Bright & 39 \\
\hline 40 & Lilienthal, C. H. & Cabinet & & $2 \mathrm{~d} N \mathrm{Y}$. & Gold & Bright & 40 \\
\hline 41 & Lilienthal, C. H. & Neptune & & $2 \mathrm{~d}$ N.Y. & Blue & Bright & 41 \\
\hline 42 & Lorillard, P. \& Co. & Bright Golden Century & & $2 \mathrm{~d} \lambda . \mathrm{Y}$. & Gold & Bright & 42 \\
\hline 43 & Lorillard, P. \& Co. & Challenge & & 2d N.Y. & Gold & Bright & 43 \\
\hline 44 & Lorillard, P. \& Co. & Century & & $2 d \mathrm{N.Y}$ & Goln & Bright & 44 \\
\hline 45 & Lorillard, P. \& Co. & Century & & $5 \operatorname{th} \times \mathrm{J}$ & Gold & Bright & 45 \\
\hline 46 & MeAlpin, D. H. \& Co. & Bright Virgin Leaf & & sd N.Y. & Gold & Bright & 46 \\
\hline 47 & MeAlpin, D. H. \& Co. & Cupid & & $3 \mathrm{~d}$ X.Y. & Gold & Bright & 47 \\
\hline 48 & MCAlpin, D. H. \& Co. & John Cornish Virgin Letef & & 3d X.Y. & Blue & Bright & 48 \\
\hline 49 & McAlpin, D. H. \& Co. & Nary & & $3 \mathrm{~d} \mathbf{X . Y}$ & Blue & Bright & 49 \\
\hline 50 & MeAlpin, D. H. \& Co. & Peach Blosson & & $3 \mathrm{~d} \mathrm{~N} Y$ & Gold & Bright & 50 \\
\hline
\end{tabular}


1 OUNC'E.

\begin{tabular}{|c|c|c|c|c|c|c|c|}
\hline No. & 1'roprietor & Brand & & Distriet & Coler & hlud of Foil & No. \\
\hline 51 & Mcalpin, D. H. \& Co. & Virgin Leaf & & $3 \mathrm{~d}$ N.Y. & Gold & Bright & 51 \\
\hline 52 & M.Bride, J. G. \& Co. & Rose Bud & & 3d Nich. & Gold & Bright & 52 \\
\hline 53 & Ml-ssinger, Charles R. & Bouquet & & 10 th Ohio & Gold & Bright & 53 \\
\hline 54 & Messinger, Charles R. & Indian Brand & & 10 th Ohio & Gold & Bright & 54 \\
\hline 55 & Miller, Mrs. G. B. \& Co. & Novelty & & Bd N.Y. & Gold & Bright & 55 \\
\hline 56 & Niller, Mrs. G. B. \& Co. & May Apple & & 3d N.Y. & Gold & Bright & 56 \\
\hline 57 & Mowry \& Co. & Nerve & & $1 \mathrm{st}$ Mich. & Gold & Bright & 57 \\
\hline 58 & Nevin \& Mills & Banner & (Small frame) & 1st Mich. & Gold & Bright & 58 \\
\hline 59 & Nevin \& Mills & Banner & (large frame) & 1st Mich. & Gold & Bright & 59 \\
\hline 60 & Parker, Holmes \& Co. & Comfort & & $1 \mathrm{st} M \mathrm{ich}$. & Gold & Bright & 60 \\
\hline 61 & Parker, Holmes \& Co. & Nerve & & 1st Mich. & Gold & Bright & $6 \mathrm{I}$ \\
\hline 62 & P'atts, Gaskin \& Patterson & Gold Leaf & & 1st Mich. & Gold & Bright & 62 \\
\hline 63 & Shotwell, D. A. \& Sons & Hero & & $2 \mathrm{~d}$ N.Y. & Gold & Bright & 63 \\
\hline 64 & Spence Bros. \& Co. & Ambrosia & & 1st Ohio & Gold & Bright & 61 \\
\hline 65 & Walker, McGraw \& Co. & Globe Chewing & & 1st Mich. & Gold & Bright & 65 \\
\hline 66 & Watts, H. H. & Tobacco Leaf & & $3 \mathrm{~d}$ N.Y. & Gold & Bright & 66 \\
\hline 67 & Williams, T. C. \& Co. & Patent Combined Plug & & $3 \mathrm{~d}$ Va. & Gold & Bright & 67 \\
\hline
\end{tabular}




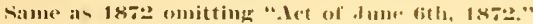

1 ( I N $\mathrm{T}$.

\begin{tabular}{|c|c|c|c|c|c|c|c|}
\hline vo. & Proprletor & Hrand & & Distriet & Coler & Kland of livil & $\sqrt{\cdots}$ \\
\hline 1 & Anderson, John \& Co. & Solace & (ents) & $32 \mathrm{~d}$ N.Y. & Gold & Bright & 1 \\
\hline 2 & Bagley, John J. \& Co. & Maytiower & & ist Mich. & Gold & Bright & 2 \\
\hline 3 & Barker, IK. C. & Anerican Eagle & & Ist Mieh. & Gold & T3right & 3 \\
\hline 4 & Barker, K. C. & Golden buxury & & Ist Xlich. & Gold & Bright & 4 \\
\hline 5 & Christman, F. \& Co. & Highland fem & & sth Ind. & Gold & Bright & 5 \\
\hline 6 & Cotterill, Fenner \& Co. & North Star & & $3 \mathrm{~d}$ Ohio & Gold & Bright & 6 \\
\hline i & Flagg, J. F. \& Co. & Seaside & & 1st N.I. & Gold & Bright & 7 \\
\hline 8 & Goodwin \& Co. & Bright Weleone & ( Light) & $32 \mathrm{~d}$ N.Y. & Gold & Bright & s \\
\hline 9 & Goodwin \& Co. & Patent & & $32 \mathrm{~d} \times \mathrm{X}$ & Gold & Bright & 9 \\
\hline 10 & Goodwin \& Co. & Welcome & (Dark) & $32 \mathrm{~d}$ X.Y. & Gold & Bright & 10 \\
\hline 11 & Goodwin \& Co. & Yellow Bank & & $32 \mathrm{~d}$ X.Y. & Gold & Bright & 11 \\
\hline 12 & Hoyt, Thos \& Co. & Nabob Chewing & & $32 \mathrm{~d}$ N.Y. & Blue & Bright & 12 \\
\hline 13 & Leggatt, Hudson \& Butler & Gilt Edge & & 1 st Mo. & Gold & Bright & 13 \\
\hline 14 & Lilienthal, C. H. & Neptune & & 2d X.Y. & Blue & Bright & 14 \\
\hline 15 & MeAlpin, D. H. \& Co. & Iohn Cornish Virgin Leaf & & 3d N.Y. & Blue & Bright & 15 \\
\hline 16 & MeAlpin, D. H. \& Co. & Navy & & $3 \mathrm{~d}$ N.Y. & Blue & Bright & I6 \\
\hline 17 & MeAlpin, D. H. \& Co. & V'irgin Leaf & & $3 \mathrm{~d}$ N.Y. & Gold & Bright & 17 \\
\hline 18 & MeAlpin, D. H. \& Co. & Peach Blossom & & $3 d X . Y$ & Gold & Bright & 18 \\
\hline 19 & Parker, Holmes \& Co. & Nerve & & 1st Mich. & Gold & Bright & 19 \\
\hline 20 & Williams, T. C. \& Co. & Patent Combined Plug & & $3 \mathrm{~d}$ Va. & Gold & Briglit & \\
\hline
\end{tabular}


$10 U N C H$.

\begin{tabular}{|c|c|c|c|c|c|c|c|}
\hline No. & Proprietor & Hrand & & Distriet & Colur & Kinal of Ioll & No. \\
\hline 1 & Allen \& Dunning & Maguolia & & 5 th N.J. & Gold & Bright & 1 \\
\hline 2 & Allen \& Ellis & Diamond & & 1st Ohio & Gold & Bright & 2 \\
\hline 3 & Allen \& Ellis & luca & & 1st Ohio & Blue & Bright & 3 \\
\hline 4 & Allen \& Ellis & Victor & & 1st Ohio & Gold & Bright & 4 \\
\hline 5 & Anderson, John \& Co. & Solace & ('Ten Cents) & $32 \mathrm{~d}$ N.Y. & Gold & Bright & 5 \\
\hline 6 & Anderson, John \& Co. & Solace & (Ten Cents) & $2 \mathrm{~d}$ N.Y. & Gold & Bright & 6 \\
\hline 7 & Anderson, John \& Co. & Solace & (For ('alifornia) & $32 \mathrm{~d}$ N.Y. & Gold & Bright & $\tau$ \\
\hline 8 & Bagley, John J. \& Co. & Gold Dust & & Ist Mich. & Gold & Buight & 8 \\
\hline 9 & Bagley, John J. \& Co. & Mayflower & & J st Mieh. & Gold & Bright & 9 \\
\hline 10 & Barker, K. C. & American Eagle & & 1 st Mljeh. & Gold & Bright & 10 \\
\hline $1 !$ & Barker, K. C. & Clipper & & Ist Mich. & Gold & Bright & 11 \\
\hline 12 & Barker, K. C. & Eureka & & Ist Mich. & Gold & Bright & 12 \\
\hline 13 & Barker, K. C. & Golden Luxury & ventis & Ist Mich. & GoliI & Bright & 13 \\
\hline 14 & Bramm, John & Virginia Leaf & & Ist N.Y. & Gold & Bright & 14 \\
\hline 15 & Buchanan \& Lyall & Acme & & 1st N.Y. & Blue & Bright & 15 \\
\hline 16 & Buchanan \& Lyall & Acme & & Ist N.Y. & Gold & Bright & 16 \\
\hline 17 & Buchanan \& Lyall & Magic & & Ist N.Y. & Gold & Bright & 17 \\
\hline 13 & Buchanan \& Lyall & Manhattan & & 1st N.Y. & Blue & Bright & 18 \\
\hline 19 & Buchner, D. & Great Central & (Name straight) & 2d N.Y. & Gold & Bright & 19 \\
\hline 20 & Buchner, D. & Great Central & (Name curved) & $3 \mathrm{~d}$ N.Y. & Gold & Bright & 20 \\
\hline 21 & Buchner, D. & Natural Leaf & (Leaf down) & 2d N.Y. & Gold & Bright & 21 \\
\hline 22 & Buchner, D. & Natural Leaf & (Leaf up) & $3 \mathrm{~d}$ N.Y. & Gold & Bright & 22 \\
\hline 23 & Buchner, D. & Sunflower & & 2d N.Y. & Gold & Bright & 23 \\
\hline 24 & Buchner, D. & Sunflower & $\ldots$ & $\therefore \mathrm{d} \times \mathrm{Y}$. & Gold & Bright & 24 \\
\hline 2.5 & Buchner, D. \& Co. & Beuefit & & $2 \mathrm{~d}$ N.Y. & Gold & Bright & $2 \pi$ \\
\hline
\end{tabular}


1 OLNCH.

\begin{tabular}{|c|c|c|c|c|c|c|c|}
\hline 10. & prentriecor & Hrand & & Mintricet & color & lintl of loull & vo. \\
\hline 26 & Buchuer, D. \& Co. & Taturaj Leaf & Leaf down & Zd X.Y. & Gold & Bright & 26 \\
\hline 27 & Campbell, Lane \& Co. & Rose of Kentlicky & & 5 th X.Y. & Gold & Bright & 27 \\
\hline 28 & Campbell, Lane \& Co. & Rose ö Kentuchy & & ith X.Y. & Gold & Bright & $2 \mathrm{~S}$ \\
\hline 29 & Catlin, D. & Golden Tireasl & & $1 \mathrm{st} M 10$. & Gold & Briglit & 29 \\
\hline 30 & Chase, lsherwood \& Co. & Buckeye & & 1utin Ohin & Gold & Bright & 30 \\
\hline $3 !$ & Cotterill, Fenner \& Co. & allen's Fine Cut & & sd Ohio & Gold & Bright & 31 \\
\hline 32 & Cotterill, Fenner \& Co. & King Bee & & 3 d Ohio & Ciold & Bright & 82 \\
\hline 33 & Cotterill, Fenner \& Co. & North Star & & 3 d Ohio & frold & Bright & 33 \\
\hline 34 & Doll \& Co. & Falls of Ohio & & 5 th $\mathrm{Ky}$. & Gold & Bright & 34 \\
\hline 3.5 & Doll \& Co. & Silver Lake & & 5 th $\mathrm{Ky}$ & Gold & Bright & 35 \\
\hline 36 & Flagg, J. F. \& Co. & Seasicle & & 1 st X.Y. & Blue & Silver & 36 \\
\hline 37 & Flagg, J. F. \& Co. & Seaside & & 1st N.Y. & Gold & Bright & $3 \pi$ \\
\hline 38 & Flagg, J. F. \& Co. & (without & t "Seven Cents") & 1 st X.Y. & Gold & Bright & 28 \\
\hline 39 & Wlagg, J. F. \& Co. & Specie & (Seven Cents) & 1st I.Y. & Gold & Bright & 39 \\
\hline 40 & Goodwin \& Co. & Best & (Darli Welcome) & $32 \mathrm{~d} X Y$. & Gold & Bright & 40 \\
\hline 4] & Goodwin \& Co. & Best & (Dark Welcome) & 2d N.Y. & Gold & Bright & 41 \\
\hline 42 & Goodwin \& Co. & Briglit Welcome & (Light Welcome) & $32 \mathrm{~d}$ N.Y. & Gold & Bright & 42 \\
\hline 43 & Goodwin \& Co. & Patent & & $32 \mathrm{~d}$ N.Y. & Gold & Bright & 43 \\
\hline 44 & Coodwin \& Co. & Plantation & & $32 d \mathrm{~N} . \mathrm{Y}$ & Blue & Bright & 44 \\
\hline $4 \bar{j}$ & Goodwin \& Co. & Plantation & & $2 d$ N.Y. & Elue & Bright & 45 \\
\hline 46 & Goodwin \& Co. & Pride of the Islant & & $2 d$ N.Y. & Blue & Silver & $4 i$ \\
\hline $4 i$ & Goodwin \& Co. & Solitaire & & $32 \mathrm{~d}$ N.X. & Gold & Bright & 47 \\
\hline 49 & Goolwin \& Co. & Welcome & ( Dark Welcome) & $32 d$ N.Y. & Gold & Bright & 4. \\
\hline 49 & Harris, A. W. \& Co. & Golden seal & & I X X.Y. & Gold & Bright & 49 \\
\hline 50 & Hofiman \& Co. & Superior & & 12th X.". & Gold & wriblit & $5 n$ \\
\hline
\end{tabular}


10 TNCE.

\begin{tabular}{|c|c|c|c|c|c|c|}
\hline No. & Propurietor & IIrand & nistrlet & Color & kint of Foll & No. \\
\hline 51 & Hoyt, Thos. \& Co. & (it lirk, front view) & $2 d$ N.Y. & Blue & Silver & 51 \\
\hline 52 & Hoyt, Thos. \& Co. & (Turk, side vlew) & $2 \mathrm{X} . \mathrm{Y}$. & Blue & Silver & 52 \\
\hline 53 & Hoyt, Thos. \& Co. & Nabob Chewing & $32 \mathrm{~d}$ N.Y. & Blue & Bright & 53 \\
\hline 54 & Irwin, A. E. & Old Friend ("Old Friend" vertical) & $2 \mathrm{~d}$ N.Y. & Gold & Bright & 54 \\
\hline 55 & Irwin, A. E. & Old Friend ("Old Friend" horizontal) & $2 \mathrm{~d} N . Y$. & Gold & Bright & 55 \\
\hline 56 & Kimball, Wm. s. & ldaho & 2Sth N.Y. & Blue & Bright & 56 \\
\hline 57 & Kimball, Wm. S. & Peerless & asth N.Y. & Gold & Bright & 57 \\
\hline 58 & Kimball, Wm. S. & Peerless Chewing & $28 \operatorname{sth}$ N.Y. & Gold & Bright & 58 \\
\hline 59 & Leggat \& Butler & Deiicious & 1 st $M 0$. & Goll & Bright & 59 \\
\hline 67 & Leggat \& Butler & Golden Era & 1 st Mo. & Gold & Bright & 6i) \\
\hline 61 & Leggatt, Hudson \& Butler & Golden Chance & lst $N 10$ & Gold & Bright & 61 \\
\hline 62 & Leggatt, Hudson \& Butler & Golden Fleece & 1 st $M o$. & Gold & Bright & 62 \\
\hline 63 & Leiderstorf, B. \& Co. & World's Fair & $1 \mathrm{st}$ Wis. & Gold & Bright & 63 \\
\hline lit & Lilienthal, C. H. & Cabinet & $2 \mathrm{~d}$ N.Y. & Gold & Bright & $6 t$ \\
\hline 6.5 & Lilienthal, C. H. & Neptnne & $2 \mathrm{~d} \mathrm{~N} . \mathrm{Y}$ & Blne & Bright & $6 \overline{5}$ \\
\hline 66 & Lilienthal, S. P. & Neptune & $2 \mathrm{~d} N . Y$ & Blue & Bright & 66 \\
\hline $6 \bar{i}$ & Lilienthal, S. P. & Young America & $2 \mathrm{~d} N . \mathrm{Y}$ & Gold & Bright & 67 \\
\hline 63 & Lorillard, P. \& Co. & Bee & 5 th N.J. & Gold & Bright & 63 \\
\hline 69 & Lorillard, P. \& Co. & Bright Golden Century & jth N.J. & Gold & Bright & 69 \\
\hline \%0 & Lorillard, P. \& Co. & Century & ith $\mathbf{N} . \mathbf{J}$. & Gold & Bright & 70 \\
\hline$i 1$ & Lorillard, P. \& Co. & Challenge & 5th N.J. & Gold & Bright & $7 t$ \\
\hline 72 & McAlpin, D. H. \& Co. & Bright Virgin Leaf & $3 \mathrm{~d}$ N.Y. & Gold & Bright & 72 \\
\hline 73 & MeAlpin, D. H. \& Co. & Cupid & $\because 1 \gg Y$ & Gold & Bright & 73 \\
\hline if & MeAlpin, D. H. \& Co. & Iohn Cornisì Virgin Leaf & :AN.Y. & Slue & Bright & 74 \\
\hline 75 & McAlpin, D. H. \& Co. & Navy & $3 \mathrm{~d} \mathrm{X} . \mathrm{Y}$ & Blne & Bright & $\pi$ \\
\hline
\end{tabular}




\section{$10 \mathrm{UNCE}$.}

\begin{tabular}{|c|c|c|c|c|c|c|c|}
\hline $\ln$. & Aroprietur & Mrsuit & & Bintriet & Aislor & tilmol \#f I oll & Yu. \\
\hline $71 ;$ & xc.Alpin, D. H. \& co. & $\backslash a y y$ & & : I XY. & Blue & Silver & $i+i$ \\
\hline 7 & Mc.Hinin, D. H. \& Co. & Peicle Blosinom & & $\because 1] \mathrm{XY}$ & fiold & Bright & $\pi$ \\
\hline 78 & AeAlpin. D. IJ. \& Co. & Virgin Letaf & & Od N.Y. & Gold & Bright & -5 \\
\hline 79 & Messinger, Charles $\mathrm{R}$. & Bouquet & & 10 th Ohio & fold & Bright & -9 \\
\hline 8) & Messinger, Charles R. & Ciranger & & Inth Ohio & Gold & Bright & So \\
\hline$\$ 1$ & Messiuger, Charlos R. & Indian Brant & & 10 th Ohio & Gold & Bright & $\rightarrow 1$ \\
\hline 82 & Meyre. 11. II. & Pilot & & Ist N.Y. & 131116 & wilver & $\therefore 2$ \\
\hline$<, 3$ & Meyer, II. 11. & Seasife & & Ist $N$. & Cinld & Bright & S:? \\
\hline 81 & Millel, Mrs. G. B. \& Co. & May Apple & (Gold) & $3 \mathrm{~d} \mathrm{~N} . \mathrm{Y}$ & (iold & Pright & ot \\
\hline 45 & Miller, Mlss, G. B. \& Co. & Mas Applr: & (Brown) & $3 \mathrm{~d}$ X.Y. & Giold & Bright & $4 . \bar{j}$ \\
\hline$\$ 6$ & Mliller, M.s. G. H. \& Co. & Prize Leif & & $\ddot{u d} \times \mathrm{X}$ & Gold & Bright & $4 i$ \\
\hline si & Nevin \& Mlills & Banner & 'with framel & 1 st $M \mathrm{ich}$. & Gold & Bright & si \\
\hline Ss & Parkfr, A. \& C'o. & Xerie & & 1sı Mich. & Gold & Brighı & b) \\
\hline 89 & Parker, Holnes \& Co. & Nerve & & Ist Mich. & Golu & Bright & $s 9$ \\
\hline (H) & Scotten, Lovet \& Co. & betroit Star No. 1 & & 1st Mich. & Gold & Bright & 90 \\
\hline 91 & Scotten, Lovett \& Co. & Hiawatha & & 1 st Xlich. & Gold & Bright & 91 \\
\hline 92 & Scotten. Lovett \& Co. & Nay Altg & & $1 \mathrm{st}$ llich. & Gold & Bright & 92 \\
\hline 93 & Senour \& Noonan & Standard Iientucky & & ith Ky. & Gold & Bright & ! \\
\hline 94 & Shotwell, D. A. \& Sons & nero & & 21 d II. Y & Gold & Bright & 94 \\
\hline $9 . ;$ & Shotwell, 1. 1. \& Sons & Lnion & & zuñ N.Y. & Gold & I3right & $9 . \bar{j}$ \\
\hline 95 & Shotwell, D. A. & Hero & & -und N.Y & Gold & Bright & 96 \\
\hline 97 & Walker, McGraw \& Co. & Globe Chewing & & ist Mich. & Gold & Bright & 97 \\
\hline 隹 & Nilliaus, T. C. E Co. & l'atent Combincel J'ug & & $\therefore d V a$. & Gold & Bright & $9 S$ \\
\hline
\end{tabular}


sime as 1875 wuitting "Serites of $1875 . "$

Tinere are 4 types of imprints of the Crooke company. Type 1 "J. J. Crooke, N.Y., Pat. Applied For." Type II "J. J. C'rooke, N Y., Foil Pat'd May 11, 1ss0." Type III "J. J. C'ochle, ‥'., l'oil l'at'd May 11, July 6, 1s80." Type IIII "The J. J. Crooke Co., N.I., Foil Pat'd May 11, July 6, 'so.'

\begin{tabular}{|c|c|c|c|c|c|c|c|c|}
\hline 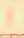 & No. & P'roprietur & irsani & & bintriet & Cotor & kind of Foil & vo. \\
\hline 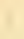 & 1 & Allen, C. W. & Lucea & . & 1st Ohio & Blue & Bright & T \\
\hline & & Anderson, John \& Co. & Solace & (Ten Cents) & $2 \mathrm{~d}$ N.Y. & Gold & Bright & 2 \\
\hline 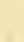 & 3 & Anderson, John \& Co. & Solace & ithout "Ten Cents") & $2 d$ N.Y. & Gold & Bright & 3 \\
\hline - & 4 & Anderson, John \& Co. & Solace & (For California) & $2 d$ N.Y. & Gold & Bright & 4 \\
\hline & 5 & Bagley, John J. \& Co. & Gold Dust & & 1 st Nich. & Gold & Bright & 5 \\
\hline 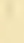 & 6 & Bagley, John J. \& Co. & Mayflower & & 1st Mich. & Gold & Bright & 6 \\
\hline & $\tau$ & Banner Tobacco Co. & Banner & (small frame) & 1st Mich. & Gold & Bright & 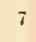 \\
\hline & 8 & Banner Tobaceo Co. & Banner & (large frame) & $\perp$ st Mich. & Gold & Bright & \\
\hline & 9 & Banner Tobacco Co. & Banner & (Imprint type 11 ) & 1 st Nich. & Blue & Rib'd Silver & \\
\hline & 10 & Barker, K. C. & American Eagle & & 1 st Mich. & Gold & Bright & 10 \\
\hline & 11 & Barker, K. C. & Eureka & & 1st Mich. & Gold & Bright & 11 \\
\hline & 12 & Beck, Aug \& Co. & Our Best & & 1 st 111. & Gold & Bright & 12 \\
\hline & 13 & Bramm, John & Virginia Leaf & & 1st N.Y. & Gold & Bright & 13 \\
\hline & 14 & Buchanan \& Lyall & Flush & & 1st N.Y. & Gold & Bright & 14 \\
\hline & 15 & Buchner, D. \& Co. & Great Central & $(1 \mathrm{mp} \cdot \operatorname{typ} I)$ & 2d N.Y. & Red & Rib'd Silver & 1 \\
\hline & 16 & Buchner, D. \& Co. & Inter-Ocean & (Imp) type l) & $2 \mathrm{IN}$ N.Y. & Red & Ril'd Silver & 16 \\
\hline & 17 & Buchner, D. \& Co. & Natural Leaf & & $2 \mathrm{~d} N . Y$. & Blue & Bright & 17 \\
\hline & 18 & Buchner, D. \& Co. & Tidal Wave & (Imp. type I) & $2 \mathrm{~d} N . Y$. & Red & Rib'd Silver & 1 \\
\hline & 19 & Campbell, Lane \& Co. & Five Cents & (Imp. type H) & 5 th N.J. & Blue & Rib'd Silver & 19 \\
\hline & 20 & Catlin Tobacco Co. & Golden Thread & & 1 st Mlo. & Gold & Bright & 20 \\
\hline & 21 & cotterill, Fenner \& Co. & King Bee & & 3d Ohio & Gold & Bright & 21 \\
\hline & 22 & Cotterill, Fenner \& Co. & Velocipede & & $3 d$ Ohio & Gold & Bright & -2 \\
\hline & 23 & Davies, IVilliam & As You Lilie it & & 1st N.Y. & Gold & Bright & $2: 3$ \\
\hline & 24 & Flint, J, G., Jr. & May Queen (Date. & $11-1-81)(1$ mprtsie 1311$)$ & 1 st Wis. & Golıl & Bright & 24 \\
\hline & $2 \pi$ & Glore, J. A. P. \& Bro's & Corn Cracker & & fith Ky, & cold & Bright & \\
\hline
\end{tabular}


1879 PROVISIONAL ISSUE (Continued) 1 1)UNCK.

FO1LS.

Sinte as 1875 Omitting "Series of $1875 . "$

There are 4 types of imprints of the crooke Company. Type I "J. J. Crooke, N.Y., Pat. Applled For." Type II "J. J Crooke, N.Y., Foll Pat'd May II, 1880." Type III "J. J. ("unke. N.Y., Foll Pat'd May II, July 6, I8so." Type IIII "."The J. J. Crooke Co., N.Y., Foil Pat'd May I1, July 6, '80,"

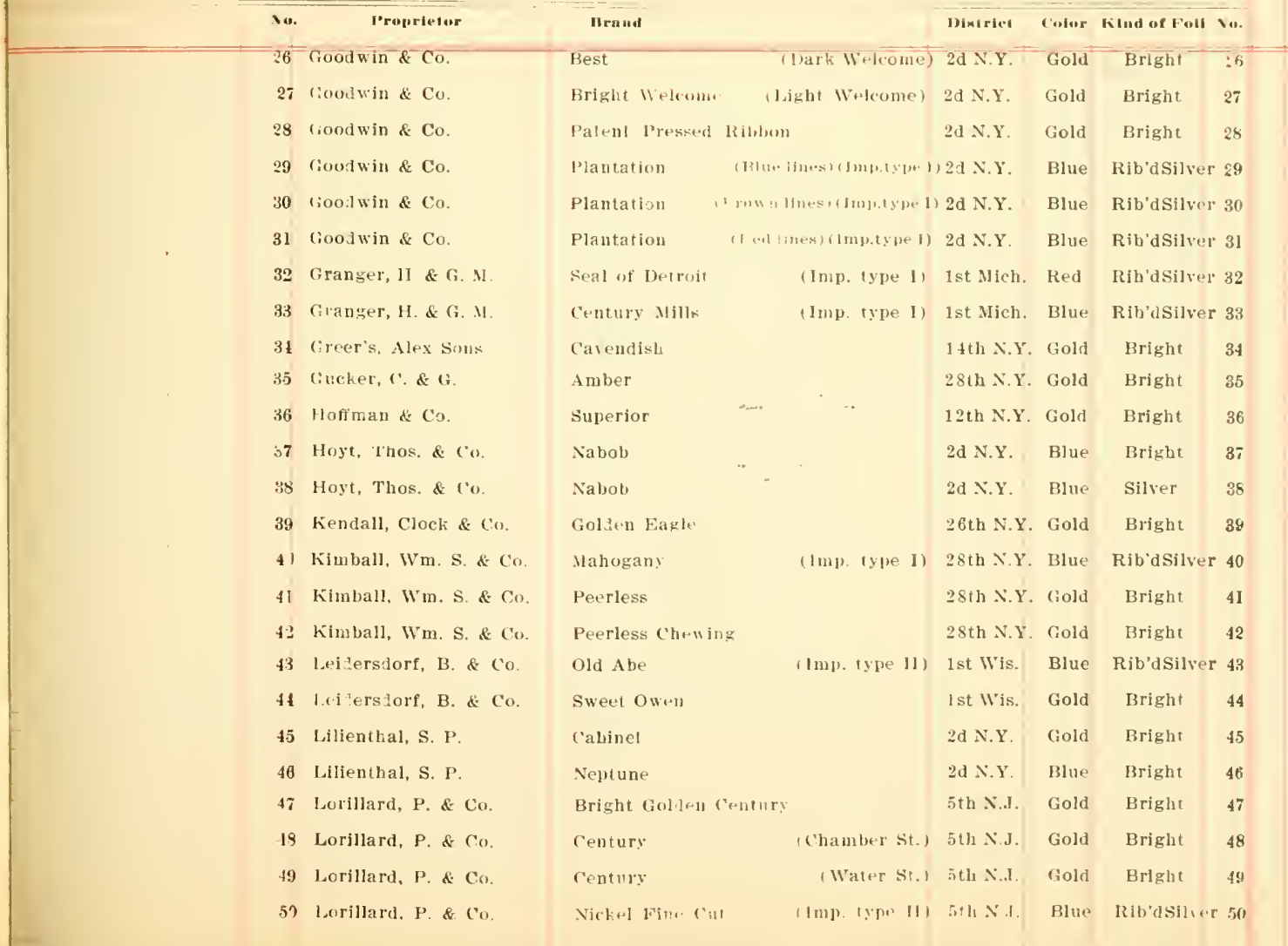




\section{Same as 1875 Onitting "Series of 187.5."}

There are 4 types of imprints of the Crooke Company. Type I "J. J. Crooke, x.l., Pat. Applied For:" Type II "J. J. Crooke, N.Y., Foll Pat'd May 11, 1880." Type III "J. J. Cruoke, X.Y.. Foil l'at'd May 11, July 6, 1880." Type IllI "The J. J. Crooke Co., N.Y., Foil Pat'd May 11, July 6, '80."

\begin{tabular}{|c|c|c|c|c|c|c|c|}
\hline$\checkmark n$. & Proprietur & Braud & & Miniried & Cowor & MInd wP latil & su. \\
\hline 51 & Lorillard, P. \& Co. & Rose Leat & . & isth N.J. & Gold & Bright & 51 \\
\hline 52 & Lorillard, P. \& Co. & Triplet & & & Gold & Bright & $5: 2$ \\
\hline 53 & Mctlpin, D. H. \& Co. & Bright Virgin Lexf & & $\therefore \mathrm{d} \times 1$. & Gold & Bright & $5: 3$ \\
\hline 54 & Medlpin, D. H. \& Co. & Cupid & & $3 d \mathrm{~N} . \mathrm{Y}$ & Gold & Bright & 54 \\
\hline 55 & McAlpin, D. H. \& Co. & Navy & & $3 d$ N.Y. & Blue & Silver & $5 n$ \\
\hline 56 & MeAlpin, D. H. \& Co. & Navy & (Im). tyne 1$)$ & $3 d$ N.Y. & Blue & Silver & 56 \\
\hline $5 \pi$ & McAlpin, D. H. \& Co. & Peach Blossom & & a N.Y. & Gold & Bright & $\bar{s}$ \\
\hline 58 & MeAlpin, D. H. \& Co. & Virgin Leaf & & $3 d \mathrm{~N} . Y$. & Gold & Bright & $5 \alpha$ \\
\hline 59 & Meyer, H. W. & Pilot & & 1 st N.Y. & Blue & Silver & 59 \\
\hline 60 & Neyer, H. W. & Pilot & (Imp) type I) & 1st N.Y. & Blue & Silver & 130 \\
\hline 61 & .1eyer, 11. W. & Seaside & & Ist X.Y. & Giold & Iright & 61 \\
\hline 62 & Miller. Mrs. G. B. \& Co. & May Apple & & $3 d$ N.Y. & Gold & Bright & 62 \\
\hline 6 is & Miller, Mls. G. B. \& Co. & Prize Leaf & $\sim \quad \sim$ & $3 \mathrm{~d} \mathrm{N.Y.}$ & Giold & Bright & 63 \\
\hline 64 & Paỹn, Benj. & colden Strawberry & & 14 th N.Y. & Gold & Bright & 64 \\
\hline 65 & Payu, Benj. & Strawberry Chewing & . . . & 14 th N.Y. & Blue & Bright & 65 \\
\hline 66 & Platts, A. H. & Golden Leaf & $\cdots$ & $26 \operatorname{th~N.Y.}$ & Gold & Bright & 6ti \\
\hline 67 & Ennour \& Noonan & Standard Kentucky & & 6 th Ky. & Gold & Bright & 67 \\
\hline 68 & Spence Bro's \& Co. & C'ash & & 1 st Ohlo & Gold & Bright & 68 \\
\hline 69 & Spence Bro's \& Co. & Wenonalı & & 1st Ohlo & Gold & Eright & 69 \\
\hline 70 & Walker, MeGraw \& Co. & Globe Chewing & & 1st Mich. & Gold & Bright & 70 \\
\hline$i 1$ & Walker, MlcGraw \& Co. & Globe Fine Cut (1:csi & n close to st.rmp, & Ist Wich. & Gold & Bright & 71 \\
\hline 72 & Walker, MeGraw \& Co. & Globe Fine Cut ceesiz & space d flom stam & 1)1 1st Mirh. & Gold & Bright & 72 \\
\hline ii: & Weighell, M. V. B. & Cavalier & & 1st Ohio & Blue & Bright & 73 \\
\hline
\end{tabular}




\section{2, 4 and 8 OUNCLS.}

There are \& types of imprints of the Crooke Company. Type 1 .J. J. Cruoke, N.Y., I’ut. Apllted For." Type If .J. J. Crooke, N.Y., Foil Pat'd May 1], J880," Type III "J. J. Cruoke, X.l.. Fofl I'at'd May 11, July ti, Jiso." Type IIII

"The J. J. Crooke Co., N.Y., Foil Pat'd May 11, July 6, '80."

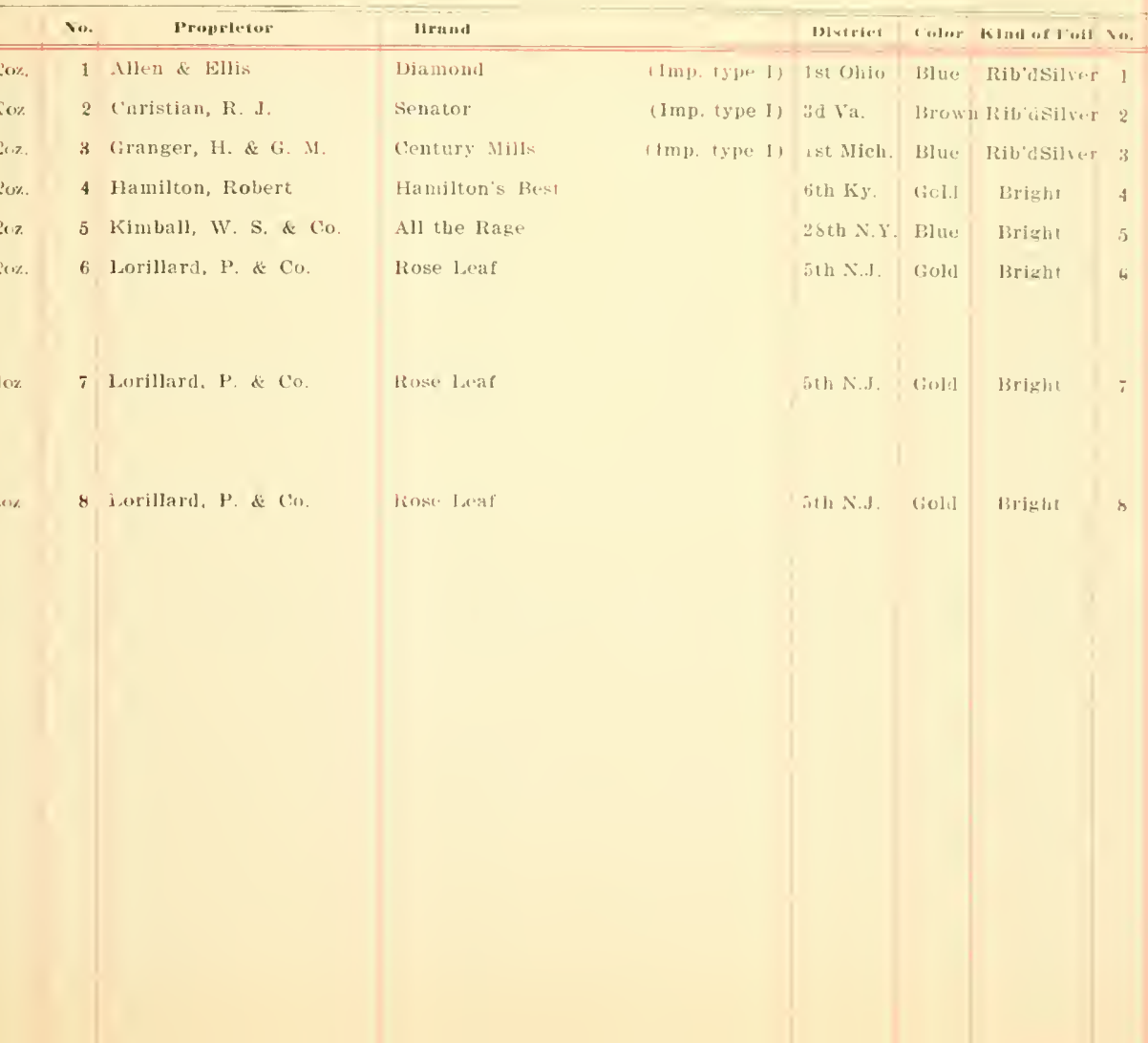




\section{UUNCE.}

There are 4 types of imprints of the Crooke Company. Type 1 "J. J. Crooke, N. $x$., Pat. Appited For." Type II "J. . Crooke, N.Y., Foil Pat'd May 11, 1880." Type III "J. J. Crooke, N.Y., Foil Pat'd May 11, July 6, 1880." Type 1llI The J. J. Crooke Co., N.X., Foll Yat'd May 11, July 6, "80."

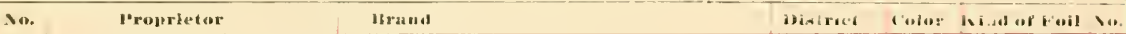

1 Mllen \& Dunning Magnoli:

2 Alleu, C. W.

Dialiond

$3-$ sen, C, W

Lucca

4 Auderson, John \& Co.

Solace

5 anderson, John \& Co.

Solace

6 Arkeuburgh, O. M. \& Co.

Aeme

7 Arkenhurgh, O. M. \& Co.

A Start

8 Arkenburgh, O. M. \& Co.

Cash

9 Alkenburgh, O. M.\& Co.

Comiqut

10 Arkenburgh, O. M. \& Co. Compass

11 Atkenburgh, O. MI. \& Co

critic

12 Arkenburgh, O. M. \& Co

Liamond

13 Arkenburgh, O. M. \& Co.

Jeannett

14 Arkenburgh, O. M. \& Co.

Maduro

15 Arkenburgh, O. M. \& Co.

Houlder's [nion

16 Arkenburgh, O. M. \& Co

Pool

17 Ackenburgh, O. M. \& Co.

Silver

18 Arkenburgh, O. M. \& Co.

19 Arkenburgh, O. M. \& Co.

20 Arkenburgh, O. $\mathrm{Ml}$ \& Co

21 Bagley, John J. \& Co.

2. Bagley, John J. \& Co.

23 Bagley, John J. \& Co.

24 Bagley, John J. \& Co.

25 Barker, K. ( . \& Co. line

West Shorr. R. M

WOII

Fast Mail

Gold Dust

MayfloweI

Sunshine tmerican Fagl.

5th N.J. Ciold Bright 1

(linp. type III, Ist OLjo Blue Ribidsilier 2

Istoijo Blue Bright 3

(Ten Cents) żd.Y. Gold Bright

for California) yd X.Y. Gola Eright 5

2u N.Y. Gold Jiright fi

¿d N.Y. Blue Bright

2d N.Y. Goli Bright s

2d X.Y. C:il Pright 9

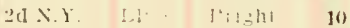

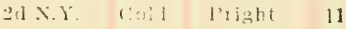

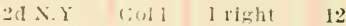

"d. Y. Ilue Bight 13

zd N.Y. Iblue Bright 14

żd N.Y. bilue Bright 15

$2 \mathrm{I}$ X.Y. Fold Pright 16

Id N.Y. Ciold Bright 17

2.I N.Y. Goll Bright Is

3.1 X.Y. Blue Bright 19

2d X.Y. (ioll Bright 20

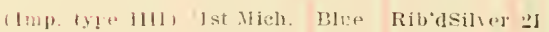

lst likh. lioll Bright 2:

1st Wich. Cioll Eright 23

ist Wich. lilue Eright it

(6. I) Hull) Ist Mich. (Goll Bright 25 
1880 ISSUE. "'Series of $1880 "$ ((')minme()).

1 OUNCE.

There are 4 types of imprints of the Crooke Company. Typ I "J. J. Crooke, N.)., I'at. Applled For." Type II "J.

J. Crooke, N.Y., Foil Pat'd May 11, 1880," Type III "J. J. Crouke, X.Y.. Foil J'at'd May 11, July b, 18xo." Type IIII "The J. J. Crooke Co., N.Y., Foil Pat'd May 11, July 6, "80,"

\begin{tabular}{|c|c|c|c|c|c|c|c|}
\hline so. & Propirleter & Uranu & & Dividing & riolur & WIud af Itoil & V... \\
\hline 26 & Barker, K. C. \& Co. & American Eagit: & (k. r Bar'er) & Ist Mich. & (ic) $1 !$ & Briz!t & $\therefore 6$ \\
\hline & Barler, k. C. \& Co. & Clipper & & lst Mith. & Gould & Pright & 27 \\
\hline 28 & Barlier, K. C. \& Co. & Eurelia & & Ist Mich. & Goll & Bright & 24 \\
\hline 29 & Eerk, Aug \& Co. & Our Best & & $1 \mathrm{st} 111$. & Golti & Briglat & 24 \\
\hline 30 & Bramm, John & Champion & & Bd X.Y. & (icll & Bright & 30 \\
\hline 31 & Bramm, John & Champion & 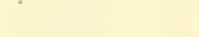 & Ist N.Y. & Goll & Bright & 31 \\
\hline 32 & Bramm, John & rirginia Leaf & & Ist X.Y. & Gol:3 & Brigl:t & $3:$ \\
\hline 33 & Buchanan \& Lyall & Dash & & Ist N.Y. & Gold & Bright & 33 \\
\hline 34 & Buchanan \& Lyall & Flush & & 1 st N.Y. & Gold & Briglit & 34 \\
\hline 35 & Buctiner, D. \& ('o. & Comique & & $2 \mathrm{~d} X \mathrm{Y}$ & Gold & Bright & 35 \\
\hline 36 & Buchner, D. \& ('o. & Natural Leaf & & $2 \mathrm{~d} N \mathrm{~K}$ & Blue & Bright & 36 \\
\hline 37 & Campbell, D. & Five Cents & (Imp. type 1111) & 5th N.J. & Blue & Kib'd Siller & $3 i$ \\
\hline 38 & Campbell, D. & Rose $\breve{o}$ Kentuck! & & 5 th N.J. & Gold & Bright & $3^{5}$ \\
\hline $3 n$ & Campbell \& Co. & Five Cents & (Inip. Iyle IIII) & 5 th . N.J. & Blife & Rib'd Silver & 39 \\
\hline 40 & Camphell \& Co. & Rose ¿ै Kentuclis & & sith X.J. & Gold & Bright & 40 \\
\hline $4 !$ & Catlin Tobacco (c). & Golden Thread & (lmp. type lisl) & Ist Mo. & Blue & Rib'd Silver & 41 \\
\hline 42 & Clark \& Snover & Anthracite & & $12 \mathrm{th}_{1} \mathrm{~Pa}$ & Gold & Bright & 42 \\
\hline 43 & ('otterill, Fenner \& (co. & North Star & & 3d Ohio & Gold & Bright & 43 \\
\hline 44 & Cotterill, Fenner \& Co. & Velocipede & & 3 d Ohis & Gold & Bright & 44 \\
\hline 45 & F:int, J. (G.. Jr. & May Queen & (Imp. Iype 1111) & 1st Wis. & Blue & Rib'd Silver & 45 \\
\hline 47 & Cail, G. W. \& Co. & Parade & & $3 \mathrm{~d}$.ld. & Gold & Bright & 46 \\
\hline 47 & Globe Tobaceo Co. & Globe & & Ist llich. & Gold & Bright & 47 \\
\hline 48 & Cranger, H. \& (i. M. & Seal of vetroit & (Imp) tyle lill) & 1st Mich. & Red & Rib'd Silver & 48 \\
\hline 49 & Gireers, Alex. Sons & Twisi Bud & & $14 \mathrm{th} \times \mathrm{X}$. & Gold & Bright & 49 \\
\hline $5 n$ & Helme, G. W. & Chic & & $3 d$ N.J. & Gold & Bright & 50 \\
\hline
\end{tabular}


1880 ISSUE. "Series of $1880 "$ (Continued).

FOI LS.

1 OUNCE.

There are 4 types of imprints of the Crooke Company. Type I "J. J. Crooke, N.Y., Pat, Applled For." Type II "J. J. Crooke, N.Y., Foil Pat'd May 11, 1880." Type III "J. J. Crooke, N.Y., Fonl Pat'd May 11, July 6, 1880." Type IIII "The J. J. Crooke Co., N.Y., Foll Pat'd May 11, July 6, '80."

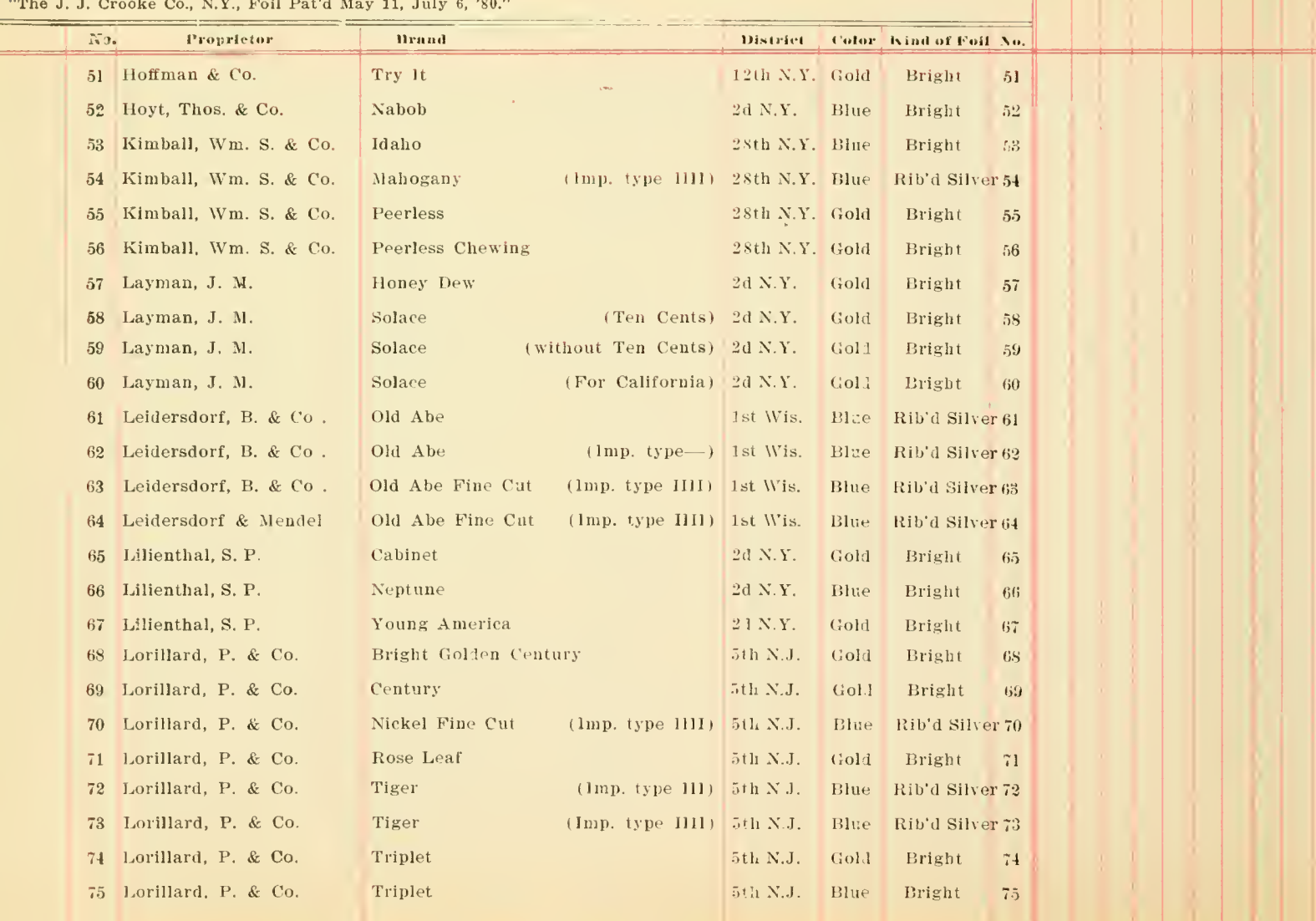


1880 ISSUE. "Series of $1880 "$ ('untinnerl).

\section{OUNCE.}

There are 4 types of imprints of the Crooke Company, Type I "J, J. Crooke, NY., Pat, Applied Fou." Tye II "J.

J. Crooke, N.Y., Foil Pat'd May 1I, IS80." Type III "J. J. Coucke, X.Y., Foll I'at'd May 11, July 6, I880." Type IIII "The J. J. Crooke Co., N.Y., Foll Pat'd May 11, July 6, "son."

\begin{tabular}{|c|c|c|c|c|c|c|c|}
\hline vo. & l'roprintor & Мrand & & Dimirlet & Color & Klist of Eou & w. \\
\hline 76 & McAlpin, 10. A. \& Co. & ('upid & & :Bl X.Y. & $c, o l d$ & Bright & $i ;$ \\
\hline$i 7$ & MeAlpin, D. H. \& Co. & John Cornish Virgin I & Leaf (whth linser) & id s.Y. & Blue & Bright & $\because z$ \\
\hline 78 & HcAlpin, D. H. \& Co. & lohn Cornish VIrgin I & Leaf (nu1nscr.) & $3 d \times r$ & Blue & Bright & $i x$ \\
\hline i9 & McAlpin, U. H. \& Co. & Lucley Fine Cut & (Imp. type IJII) & :Id XY. & Iilue & Rib'al silver & $r y$ \\
\hline so & McAlpin, 1). H. \& Co. & Nay & (lmp. type l!l) & $3 \mathrm{~d}$ N.Y. & Blue & Rib'd Silver & $r s 0$ \\
\hline$\$ 1$ & Medluin, U. il. \& Co. & Nary & (Inp). type 11II) & $3 \mathrm{~d}$ N.Y. & Blue & Rila'd Silver & r \$I \\
\hline$\varepsilon 2$ & .leAlpin, D. H. \& Co. & Navy & (No Imp.) & Z̈ X.Y. & Elise & Bright & 82 \\
\hline$\varsigma_{3}$ & MeAlpin, D. H. \& Co. & Nary & (lmp) type H11) & $3 \mathrm{~d}$ x.Y. & mue & Blight & 83 \\
\hline 84 & aleAlpin, I). H. \& $\mathrm{Co}$. & Peach Blossom & & :a x.I. & Ciola & Brigbt & $\delta 4$ \\
\hline 85 & McAlpin, D. H. \& Co. & l'irgin Leaf & with inseription) & $\because \mathrm{d} N$ N.Y. & Giold & Bright & 8.5 \\
\hline$\because 6$ & McAlpin, D. H. \& Co. & Virgin Leaf & hout inseription) & $3 \mathrm{~d}$ X.Y. & Giold & Bright & 86 \\
\hline 87 & Meyer, H. W. & Gold Leaf & & Ist X.Y. & Gold & Bright & 87 \\
\hline 88 & Neyer, H. W. & Pilot & (Imp. type IIII) & 1st N.Y. & Blue & Rib'd Silver & 88 \\
\hline 89 & Heyer, H. W. & Sun & & 1st N.Y. & Gold & Bright & 89 \\
\hline 10 & Miller, Mrs. G. B. \& Co. & May Apple & & 3d N.Y. & Gold & Bright & 98 \\
\hline 91 & Miller, Mrs. G. B. \& Co. & Prize Leaf & & $3 \mathrm{U}$ N.Y. & Gold & Bright & 91 \\
\hline 92 & Palmer, A. N. \& Co. & Chink & & 24 th N.Y. & Red & Bright & 12 \\
\hline 93 & Palmer, A. N. \& Co. & Chimney Cormer & (Imp. type 111I) & 24 th N.Y. & Hlue & Rib'd Silver & $\mathrm{r} 93$ \\
\hline 94 & Pnyn's, B. Sons & Golden Strawberry & & 14th N.Y. & Gold & Brigbt & 94 \\
\hline 9.5 & Pryn't, B. Sons & Strawberry Chewing & & [4th X.Y. & i3lue & Bright & 95 \\
\hline 6 & Platts, A. H. \& Co. & Gold Leaf & & -6fth X.Y. & Gold & Bright & 96 \\
\hline 97 & Rosenfeld, E. & Temptation & & ISth Ohio & Gold & Bright & 97 \\
\hline 98 & Srhriber, J. \& Co. & Prairie Flower & & isth Obio & Gold & Bright & $\$ 8$ \\
\hline 97 & Shields, Francis & Sweet Cavendish & & Ifth N.X. & Gold & Bright & 99 \\
\hline 100 & Spence Bros. \& Co. & Cash & & Ist Ohio & riold & Bright & 101 \\
\hline
\end{tabular}




\section{OUNCES.}

Tliere are 4 types of imprints of the Crooke Company. Type 1 "J. J. "rooke, X.Y.. l'at. Applict For." Type II "J. J. Frooke, N.Y., Foil Pat'd May 11, 1850," Type III "J. J. C rooke, N.Y., Foil I'at'd May 11, JuIy 6, 1580." Type IllI "'The J. J. Crooke Co, N.Y., Foil Pat'd May 11, July 6, 'so."

\begin{tabular}{|c|c|c|c|c|c|c|}
\hline No. & Proprietor & Hrand & Dintriet & Coior & Kind of Foil & No. \\
\hline 1 & Allen, C. W. & (Chicago) (Imp.type Illf) & 1st III. & Blue & Rib'd Silver & 1 \\
\hline 2 & Allen, C. W. & (Cincinnati) (Imp,type IIII) & 1st Ohio & Blue & Rib'd Silver & 2 \\
\hline 3 & Arkenburgh, O. M. \& Co. & (with "M. H. Ricders") & $2 \mathrm{~d} \mathrm{N.Y.}$ & Blue & Bright & 3 \\
\hline 4 & A:kenburgh, O. M. \& Co. & (witbout "M. H. Rieders") & 2d N.Y. & Blue & Bright & 1 \\
\hline 5 & Arkenburgh, O. M. \& Co. & Bang & $2 \mathrm{~d}$ N.Y. & Blue & Brigbt & 5 \\
\hline 6 & A: kenburgh, O. Ml. \& Co. & Dark Shorts & $2 d$ N.Y. & Blue & Bright & 6 \\
\hline 7 & Arkenburgh, O. M. \& Co. & Fireside & $2 \mathrm{~d}$ N.Y. & Blue & Bright & i \\
\hline 8 & Arkenburgh, O. M. \& Co. & Society & $2 \mathrm{~d}$ N.Y. & BItie & Bright & 8 \\
\hline 9 & Al kenburgh, O. M. \& Co. & United States & $2 d \mathrm{~N} . Y$. & Blue & Bright & 9 \\
\hline 10 & Hamilton, Robert & Hamilton's Best & 6th N.Y. & Gold & Bright & 10 \\
\hline 11 & Kimball. W. S. \& Co. & Mahogany Cut Plug & 28 th N.Y. & Blue & Bright & 11 \\
\hline 12 & Kimball, W. S. \& Co. & Mahogany Cut Plug (Imp. type III) & $2 S \operatorname{th} N . Y$. & Blue & Rib'd Sllver & \\
\hline 13 & Inmball, W. S. \& Co. & Mabogany Cut Plug (Imp. type IIII) & 28 th N.Y. & Blue & Rib'd Silver & 13 \\
\hline 14 & Leidersdorf, B. \& Co. & Old Abe Fine Cut & 1st Wis. & Blue & Rib'd Silver & 14 \\
\hline 15 & Leidersdorf \& Mendel & Old Abe Fine Cut & 1 st Wis. & Blue & Rib'd Silver & \\
\hline
\end{tabular}


1883 ISSUE. '“Series of 1883.'

\section{1 ()UNCE.}

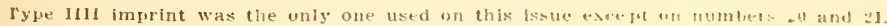

\begin{tabular}{|c|c|c|c|c|c|c|c|}
\hline & s.. & proprietur & Urnat & Dintrlet & alor & In In] of I inll & No. \\
\hline \multirow{7}{*}{ ' } & & Allen \& Dunning & Home Rulettion Hult in colorless letters & s) $5 \operatorname{th} \mathrm{N} . \mathrm{J}$ & Gold & Briglıt & 1 \\
\hline & 2 & Allen \& Dunning & 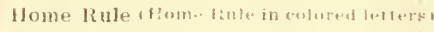 & I.th N.J. & Golll & Brigl, & 2 \\
\hline & 3 & Illen \& lounning & Magnolia & ith $\mathrm{X} . .1$ & fiolit & Hright t & 3 \\
\hline & 1 & Anericair Eagle Tob. Co. & Anerican Eagle & Ist Mich. & Golll & Brig! i & 4 \\
\hline & $\bar{j}$ & American Eagle Tob. Co. & Eureka & Ist Mich. & Gold & Briglit & 5 \\
\hline & $\mathbf{6}$ & Anderson, John \& Co. & ("Five ("nuts") & $2 \mathrm{~d} \times \mathrm{Y}$ & Gold & Briglat & $\mathrm{ti}$ \\
\hline & 7 & Anderson, John \& Co. & Honey Dew (without "Five Cents") & $2 \mathrm{~d}$ ×. Y. & Gold & Bright & 7 \\
\hline \multirow[t]{18}{*}{$\cdot$} & 8 & Anderson, John \& Co & (Ten Cents) & $\because \mathrm{d}$ N.Y. & Gold & Bright & 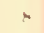 \\
\hline & $y$ & Anderson, John \& Co. & "without "Ten Cents") & $2 \mathrm{~d} \times \mathrm{Y}$ & liold & Briglt & $\theta$ \\
\hline & 10 & Auderson, John \& Co. & For ('alifernia) & $2 d X Y$ & Gold & Bright. & 10) \\
\hline & 11 & Ankerburgh, O. M. \& Co. & A Start & $21 \mathrm{N.Y}$ & Blue & Bright & 11 \\
\hline & 12 & Arkenburgh, O. M. \& Co. & Cash & $21 \mathrm{VI}$ & Gold & Bright & 12 \\
\hline & 13 & Arkenburgh, O. II. \& Co. & C. O. I). & $2 \mathrm{i} \times \mathrm{K}$. & Gold - & Bright & 13 \\
\hline & 14 & Arlienburgh, O. M. \& Co. & C. O. D. & $2 \mathrm{~d}$ N.Y. & Blue & Bright & 14 \\
\hline & 15 & Arkeaburgh, O. M. d Co. & Compass & $2.1 \times 1$. & Blue & Briglit & 15 \\
\hline & 16 & Arkenburgh, O. M. \& Co. & Critic & $2 \mathrm{ll}$. K. Y. & Gold & Briglit & 16 \\
\hline & 17 & Arkenburgh, O. $11 . \&$ Co. & Delightful & $2 \mathrm{~d} N \mathrm{Y}$ & Gold & Bright & 17 \\
\hline & 18 & A"lienburgh, O. M \& Co. & Delightful & $2 d X Y$ & Blue & Bright & 18 \\
\hline & 19 & Ailieuburgh, O. M. \& Co. & Diamond & $2 \mathrm{~d} N \mathrm{~V}$ & Gold & Bright & 19 \\
\hline & 20 & Arkenburgh, O. MI. \& Co. & 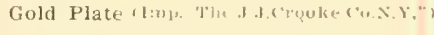 & $12 d x . Y$ & Blue & Bronze & $£ 0$ \\
\hline & 21 & Arke,dburgh, O. . I. \& Co. & 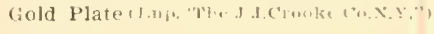 & $2 \mathrm{~d}$ X.Y. & Red & Bronze & 21 \\
\hline & 22 & I'henburgh, O. M. \& Co. & Inlaid & $2 \mathrm{~d}$ N.Y. & Gold & Bright & 32 \\
\hline & 23 & A:kenburgh, U. M. \& Cu. & Maduro & $2 d X Y$ & Blue & Bright & 23 \\
\hline & 2 & B:hasb.rgh, O. M. \& C & Moulder's Union & $21 \mathrm{~N} . \mathrm{Y}$ & Blue & Bright & 24 \\
\hline & 25 & Arkinburgh, O. MI. \& Co. & silver & $2 \mathrm{~d}$ N.Y. & Gold & Brigbt & 25 \\
\hline
\end{tabular}


1 OUNCE.

I'ype IIII imprint was the only one used on this issue except on आumlurs 20 and 21.

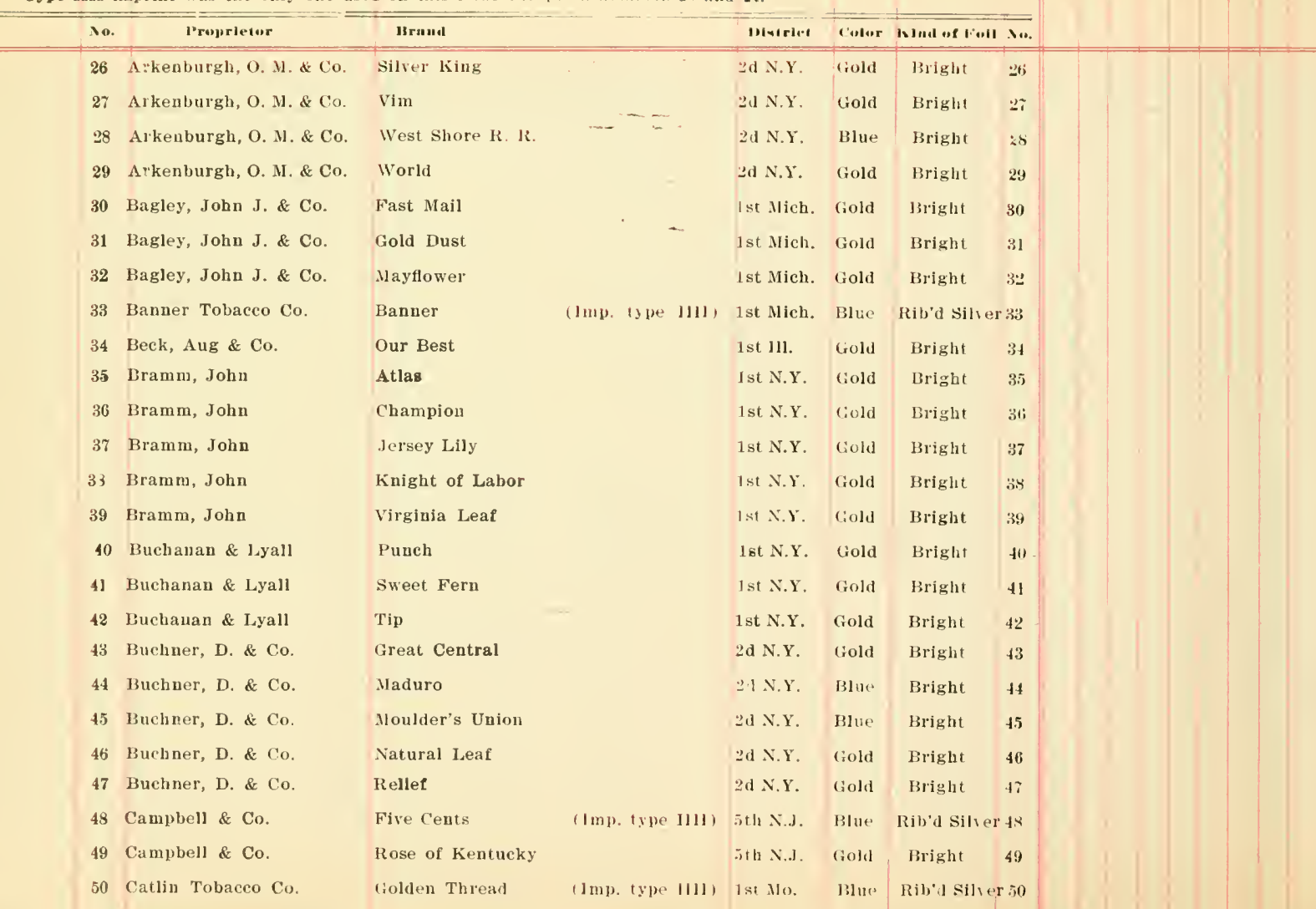




\section{$10 \mathrm{ONCH}$.}

Type 1111 imprint was the only one uses on this issue exent nu numbers 20 and 21.

\begin{tabular}{|c|c|c|c|c|c|c|c|}
\hline$\checkmark$. & Propipletor & "Irnn! & & Dintriet & Color & KJ ad of Foll & lo. \\
\hline 53 & Cotterill, Fenner \& Co. & North Star & & 3d Ohio & Gold & Bright & il \\
\hline 52 & Cotrerill, Fenner \& $\mathrm{Co}$. & North Star & & J st Ohio & Gold & Bright & 52 \\
\hline 5.3 & Cotterill, Fenner \& Co. & Velocipede & & 3d Ohio & Gold & Bright & 53 \\
\hline $5 t$ & Crawford, J. A. \& Soms & Famous & & 6 th Ky. & Gold & Bright & 54 \\
\hline $5 \overline{5}$ & Crawford, J. A. \& sons & Old (iold & & 6th Ky. & Gold & Bright & 35 \\
\hline 56 & F'int, J. G., Jr. & May Queen & (1mi). 19pe 1311) & $1 \mathrm{st} W$ is. & Blue & Rib'd Silver & r 56 \\
\hline 57 & Frishmuth Bros. \& ('o. & Luxury & & Ist Pa. & Gold & Bright & 57 \\
\hline 58 & fiail \& Ax, G. WV. & Parate & & $3 d \mathrm{~d} d$ & Gold & Bright & 58 \\
\hline 59 & Globe Tobaceo Co. & Broadway & & Ist Nich. & Gold & Bright & 69 \\
\hline 6i0 & Globe Tobacco Co. & Ed de C's Best & & Ist Mich. & Gold & Bright & 60 \\
\hline 61 & Globe Tobaceo Co. & Globe & & Ist Nich. & Gold & Bright & 61 \\
\hline (i) & Globe Tobacea Co. & Hope & & Ist Mich. & Gold & Bright & 62 \\
\hline 68 & Greer's, Alex. Sons & Royal & & 14th N.Y. & Gold & Bright & 63 \\
\hline 64 & Hamilton, Robt. & $x x$ & & $6 \mathrm{th} \mathrm{Ky}$. & Gold & Bright & 64 \\
\hline 65 & Hamilton, Robt. & $x \times$ & & 6 th $\mathrm{ky}$. & Blue & Rib’d Silver & 65 \\
\hline 66 & Helme, G. W. & Chic & & $3 \mathrm{~d}$ N.Y. & Gold & Bright & 66 \\
\hline 67 & Herbs, F. \& M. & Diploma & & 12 th N.Y. & Gold & Bright & 67 \\
\hline 68 & Hoffman \& Co. & Try lt & & 1 2 th N.Y. & Gold & Bright & $6 s$ \\
\hline 69 & Hoffman \& Co. & Try lt & & 15th N.Y. & Gold & Bright & 69 \\
\hline 70 & Kimball, W. S. \& Co. & klaho & & 28th N.Y. & Blue & Bright & 70 \\
\hline 71 & Kimball, W. S. \& Co. & Nahogany & & 2Sth N.Y. & Blue & Rib'd Silver & 71 \\
\hline 72 & Kimball, W. S. \& Co. & Peerless & & 2sth N.Y. & Gold & Bright & 72 \\
\hline 73 & Kimball, W. S. \& Co. & Peerless Chewing & & 28th N.Y. & Gold & Bright & 73 \\
\hline it & Kimball. W. S \& Co. & Star & & $28 \operatorname{th}$ N.Y. & Gold & Brigbt & i4 \\
\hline 75 & Laynan, I. M. & Honey Dew & ( Fise (ents) & $21 \mathrm{~N} . \mathrm{Y}$ & fiold & Bright & 75 \\
\hline
\end{tabular}


1 OUNCE.

Type IIII Imprint was the only one used on this issue except min rumbers 20 and 21.

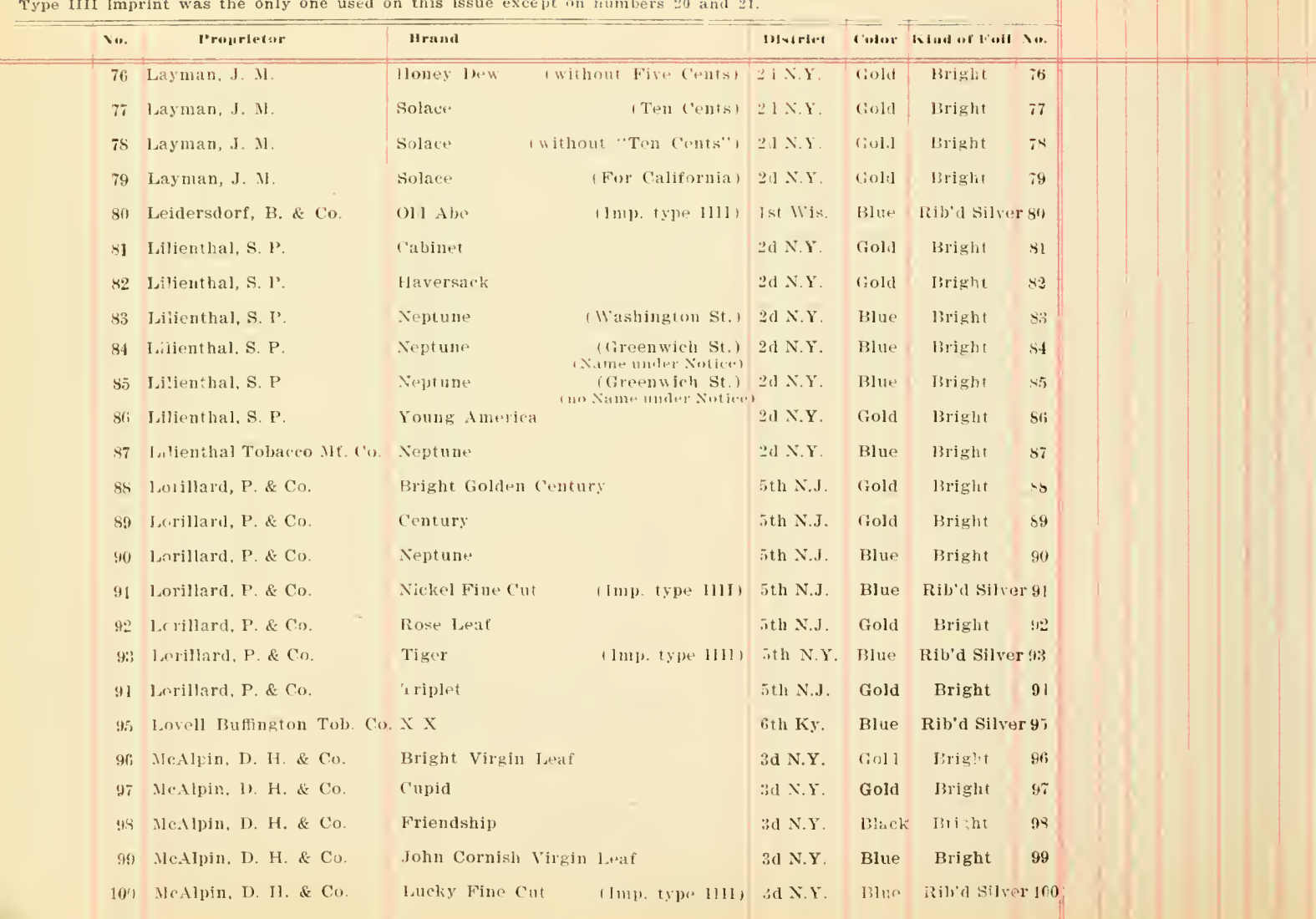


1883 ISSUE. "'Series of 1883 "' (Continued).

\section{UNNCL:}

Type IIII imprint was the only one used on this issue txiept on numbers 20 and 21 .

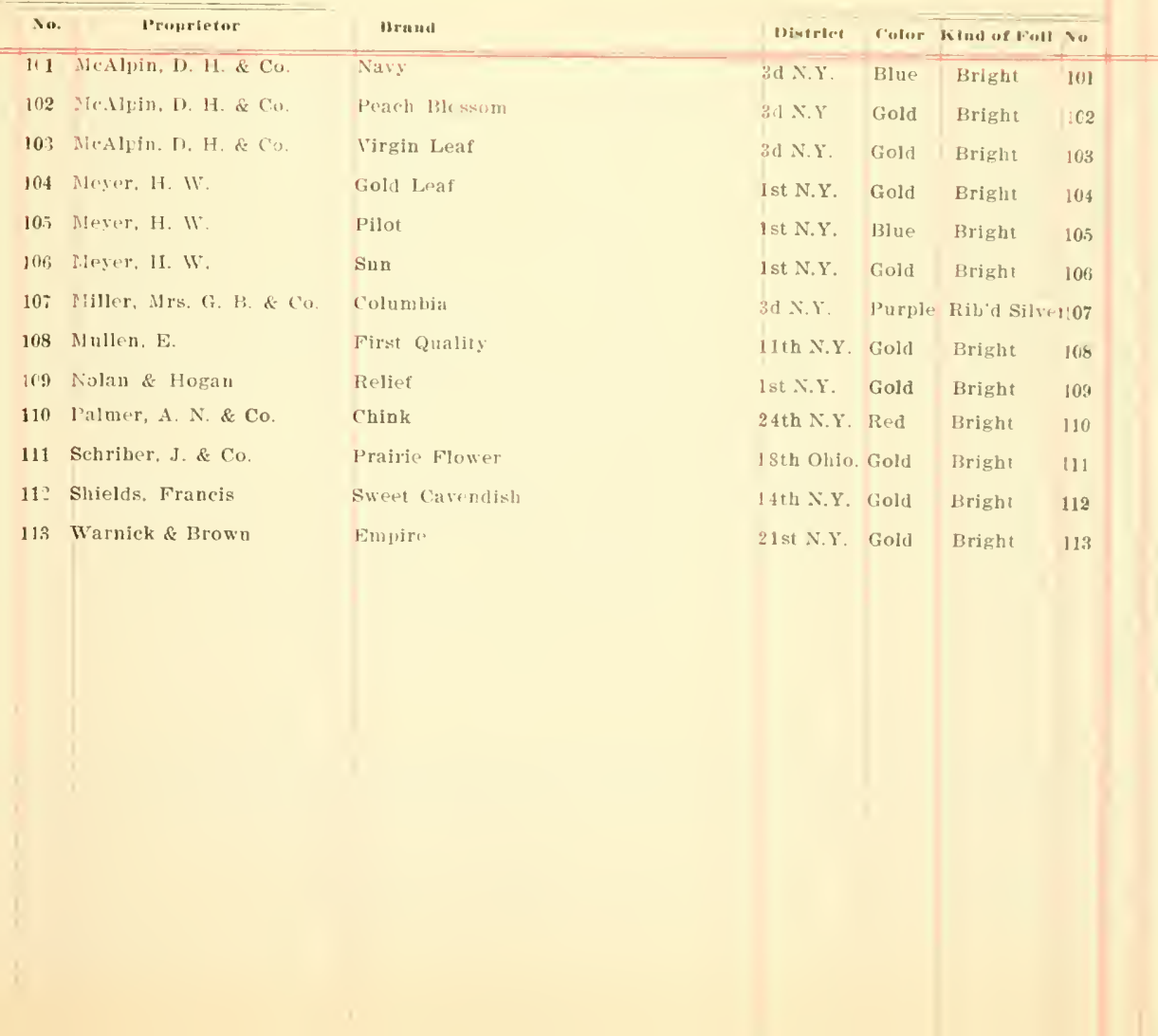


2,4 and 8 OUNCES.

Type IIII imprlnt was the only one used on this issue.

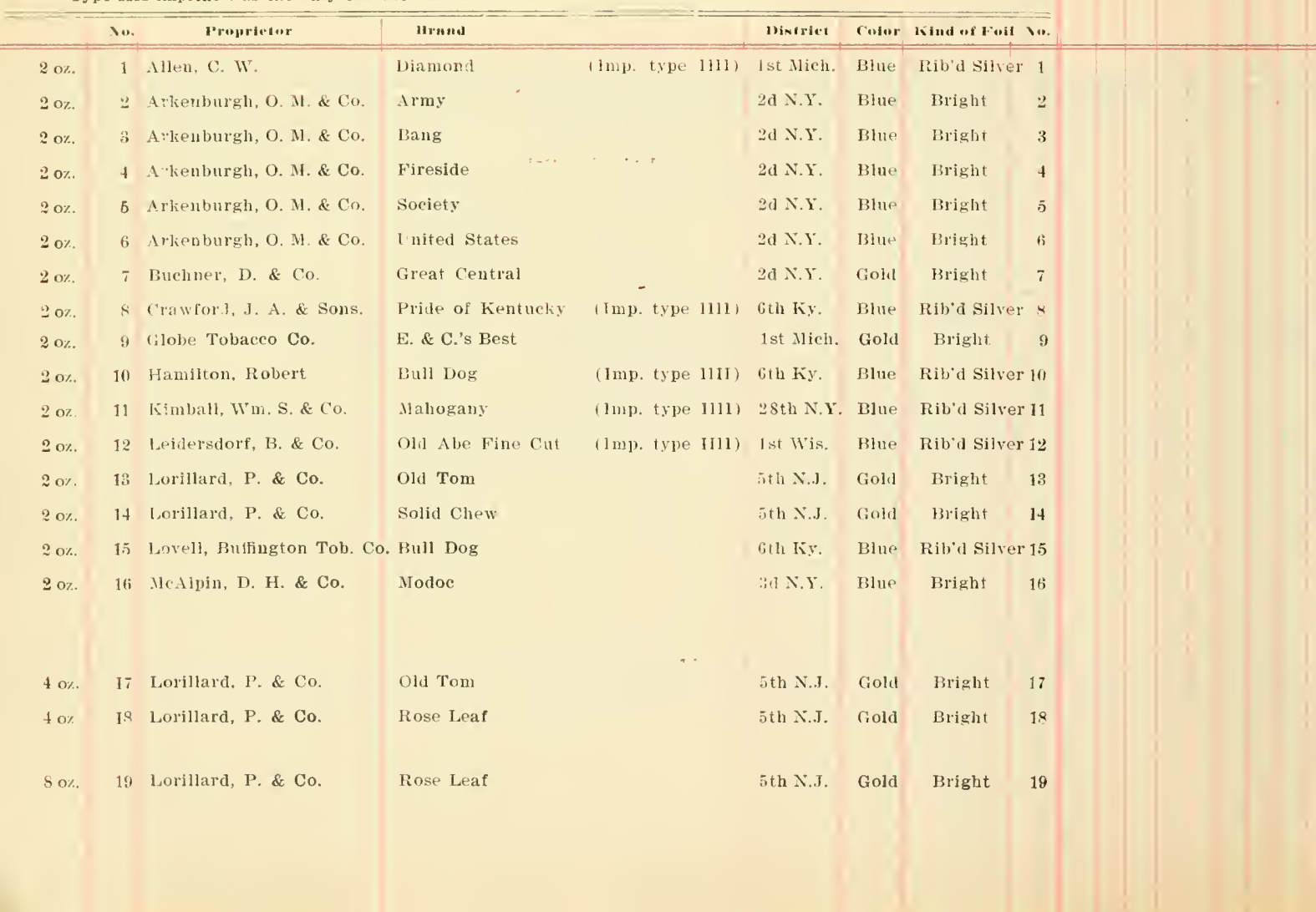




\section{$10 \mathrm{TNCH}$}

Type llt imprint was the only one used on this issut.

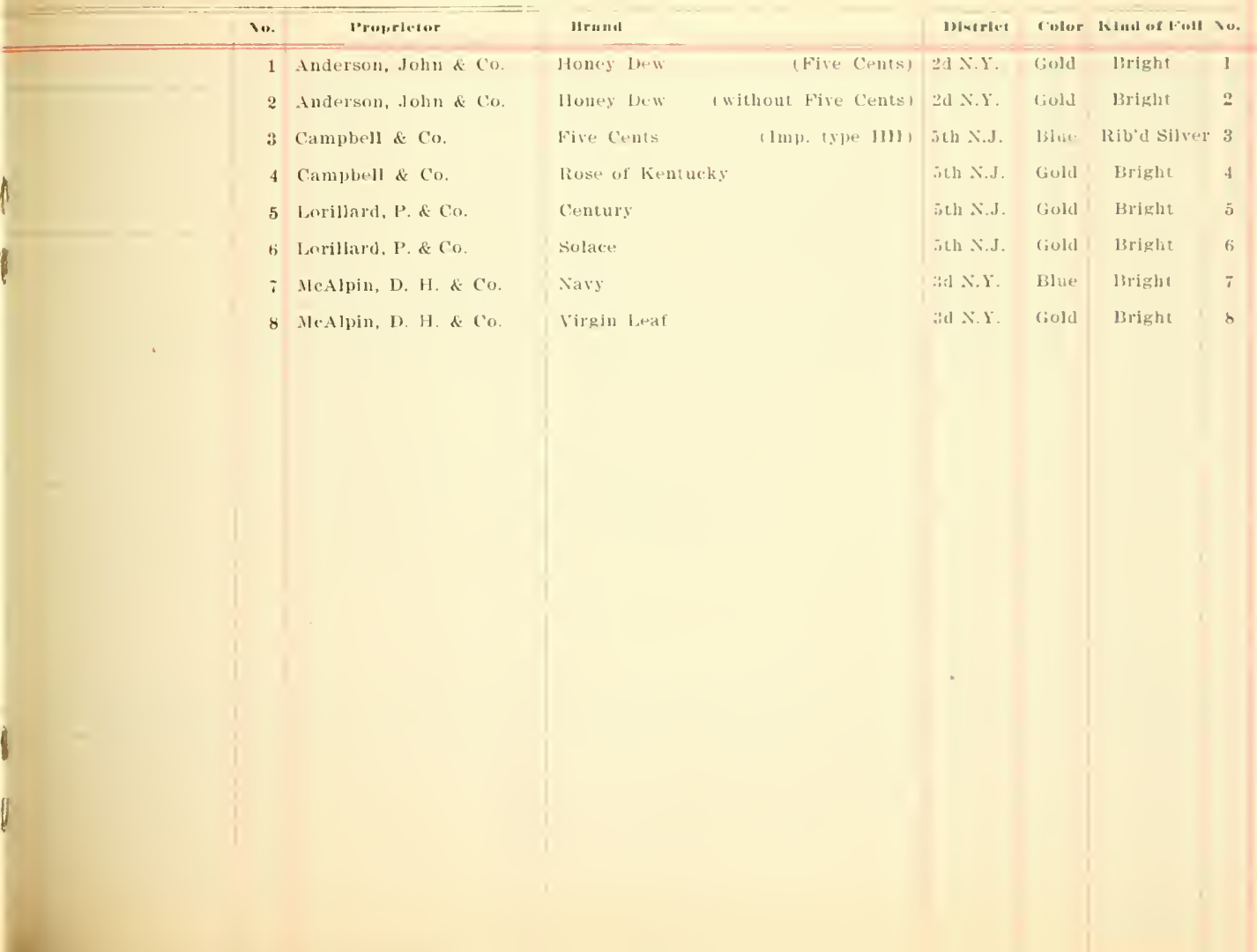




\section{OUNCE.}

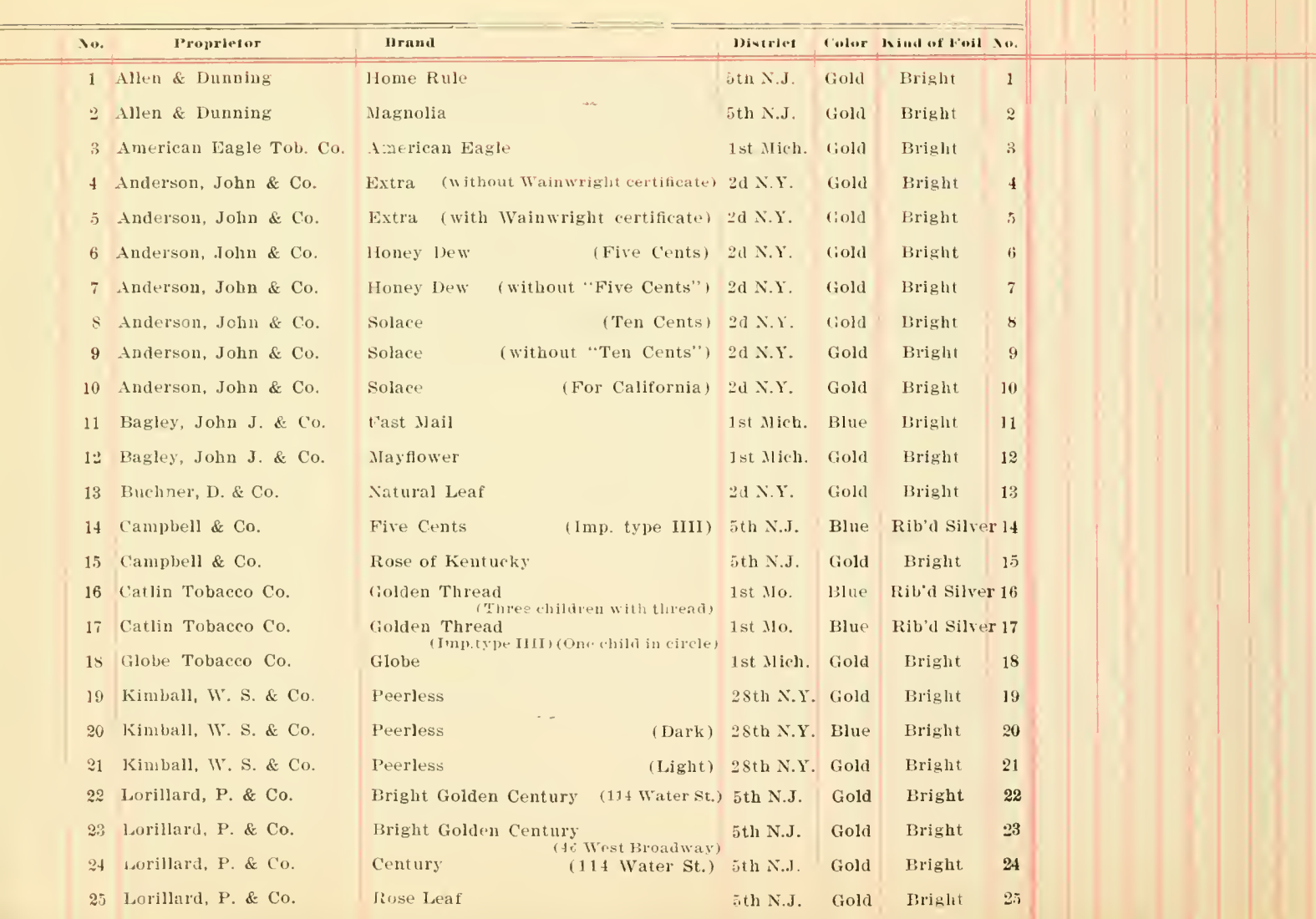




\section{$10 \mathrm{UNC} \mathrm{F}$.}

\begin{tabular}{|c|c|c|c|c|c|c|c|}
\hline vo. & I'roprletur & Irand & & Minirlet & rosiur & wind ue loull & las. \\
\hline 26 & Lorillard, P. \& C & Tiger & $(\operatorname{lmp} . t y p e=1111)$ & 5th N..1. & Hlue & Rib'd Silver & 26 \\
\hline 27 & Lorillard, P. \& Co. & Triplet & & 5 th X.J. & Gold & Brlght & 27 \\
\hline 28 & Lorillard, P. Company & ('entury & West Broadway, & ith X..1. & Gold & Bright & 28 \\
\hline 29 & Lorillard. P. Company & century & III Hudson, & ith N.J. & Gold & Bright & 29 \\
\hline 30 & Lorillard, P. Company & Veptunt & & ith X.J. & IBlue & l’rlght & 30 \\
\hline 31 & Lorillaro, P. Company & 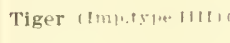 & | 11: West I:roadwar, & ith X.J. & mue & RII,d sllver & 31 \\
\hline 32 & Lorillard. P. Company & Triplet & & ith X.J. & Golil & lirlght & 32 \\
\hline 33 & Lovel] Buftington Tob. Co. & Bull loge & (no Imprint) & 6th Ky. & Blue & Rilod Silver & r 33 \\
\hline 34 & McAlpin. D. H. \& Co. & Navy & & $\because$ d ト. & lilue & Bright & 34 \\
\hline 35 & MrAlpin, D. H. \& Co. & Pearcll lalossona & & 31 N.Y. & Gold & Brigh & 3.5 \\
\hline 36 & MeAlpin. D. H. \& $\mathrm{Co}$. & Virgin Leaf & & $3 d \mathrm{~N} . \mathrm{Y}$ & (iold] & Bright & 36 \\
\hline 37 & Shields \& Sons & Sweet Carcudisla & & 1 th $\times Y$. & Gold & Bright & 37 \\
\hline 38 & IT. S. Tobaceo Co. & Saratogd & & $2 \mathrm{~d} \mathrm{Va}$ & Goles & Bright & 38 \\
\hline
\end{tabular}


1890 ISSUE. "Act of Oct. 1, 1890."

2 OUNCES.

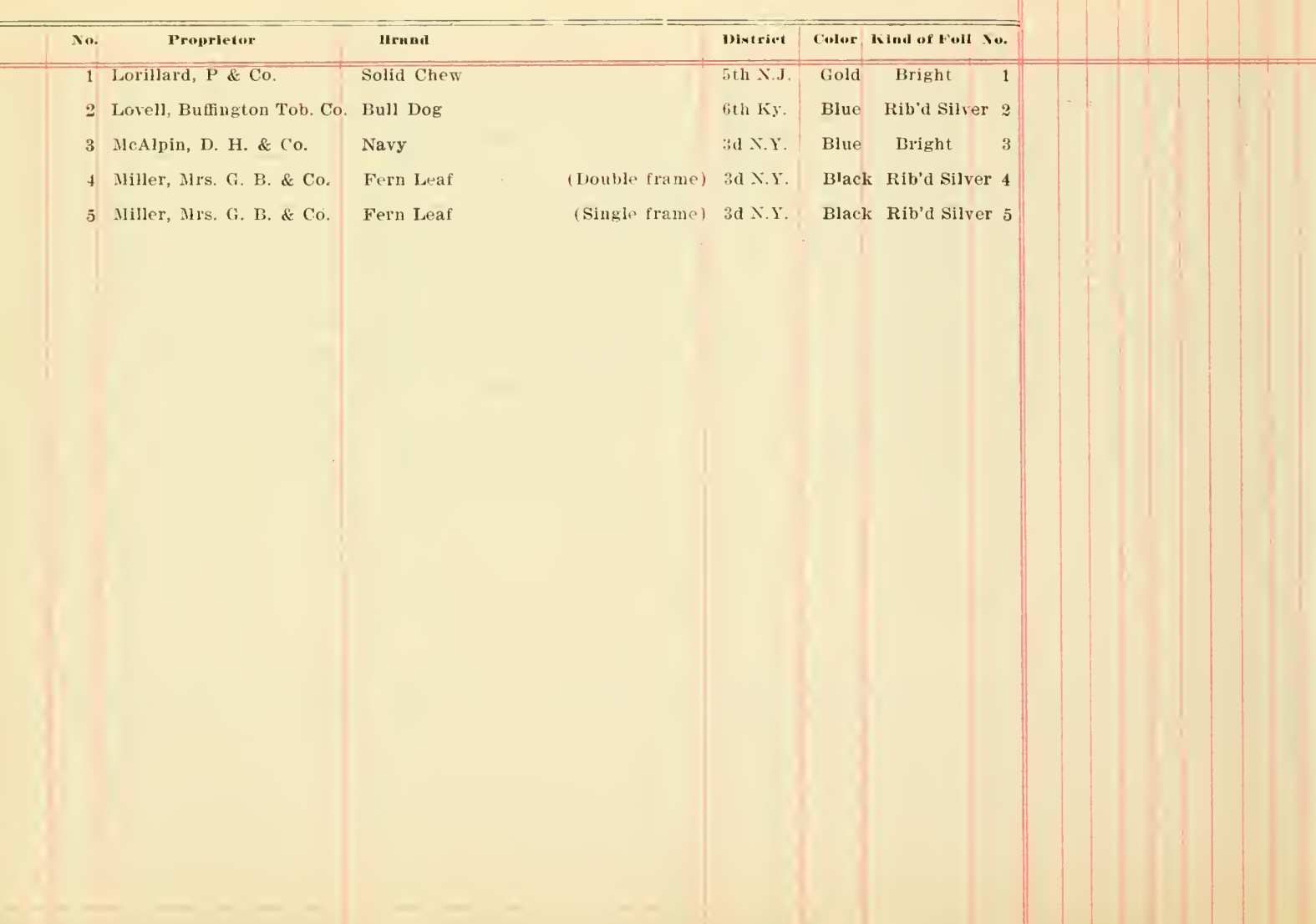


Same as 1890 Omitting "Act of (bet. 1, 1890."

1 OUNCE.

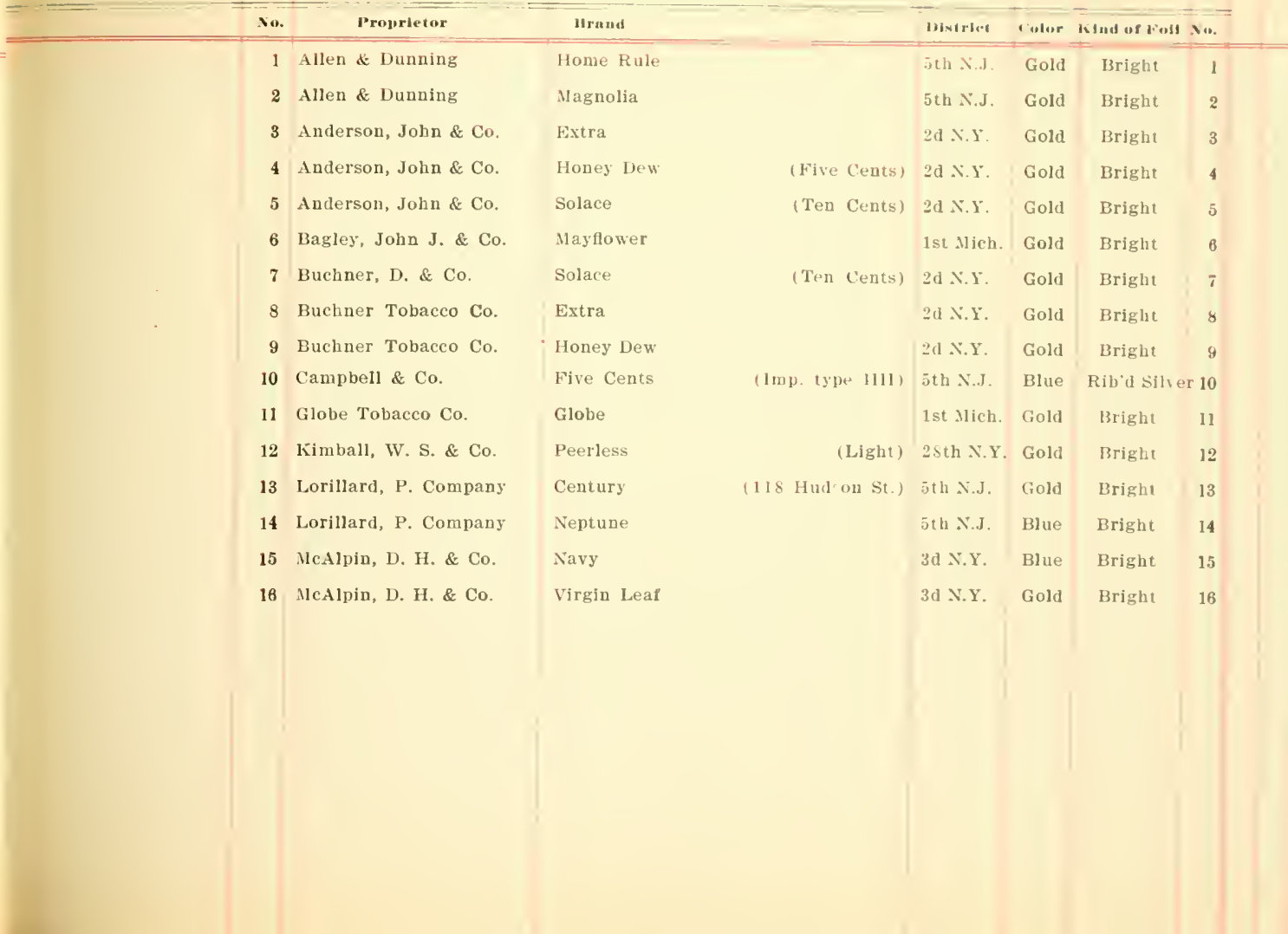


Sinte as $18 ! 00$ Omitting "Act of bet. 1, 1890" and value.

1 2-3U OTNCFS.

AT BOT'TOM.

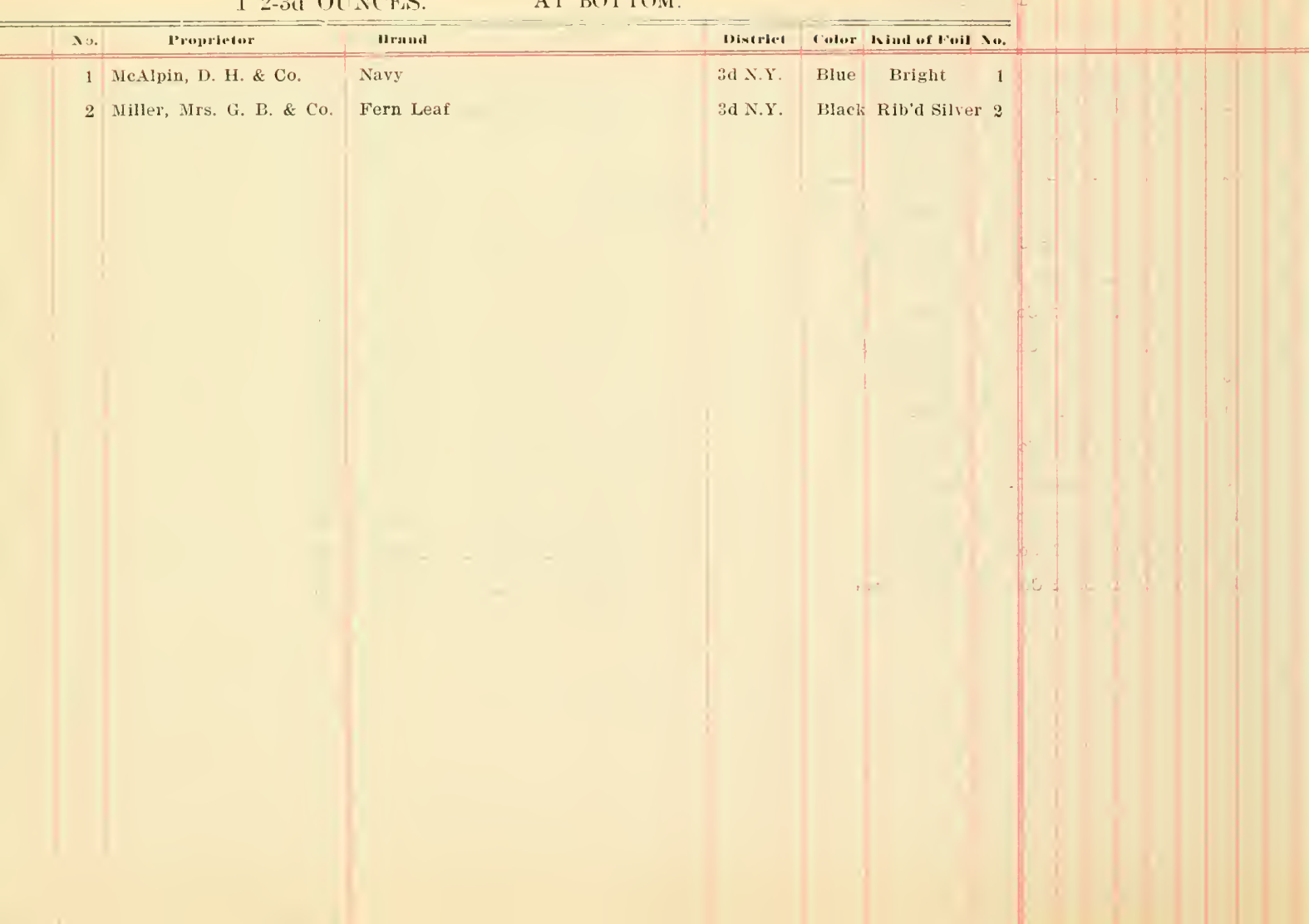


1898 ISSUE. "Act of June 13, 1898."

1 OUNCE.

\begin{tabular}{|c|c|c|c|c|c|c|c|}
\hline u. & Propirtetor & Mrond & & Dintrlet & Culor & Kind of rous & $\sqrt{10 .}$ \\
\hline 1 & American Tosacco Co. & Sclace & & & Gold & Bright & 1 \\
\hline 2 & Anerican Tobacco ('o. & Virgin Leaf & & & liold & Bright & 2 \\
\hline$:$ & Bagley, John J. \& Co. & May flower & & 1 st .lich. & Giold & Bright & 3 \\
\hline 4 & Buchner Tobacco Co. & Solace & & $2 \mathrm{~d} \times \mathrm{X}$. & Giold & Bright & 4 \\
\hline j & Campbell Tobaceo Co. & Five Cents & Im1). type 1111) & ith $\times$.J. & Lilue & Rib'd Silver & 5 \\
\hline 6 & Campbell Tobacco Co. & Ciolden Rod & & 5 th X.J. & Blue & Gold & 6 \\
\hline$i$ & Globe Tobaceo Co. & Globe & & 1st Mich. & Giold & Briglit & $i$ \\
\hline 8 & Hoffman \& Co. & Red Hook Extra & & & ked & Bright & s \\
\hline 9 & Kimball, W. S. \& Co. & Peerless & (Light) & & Gold & Briglit & $y$ \\
\hline 10 & Lorillard, P. Company & ( entury & ( I s Hudson st.) & sth N.J. & Giold & Bright & 111 \\
\hline 11 & Lorillard, P. Company & C'entury & (111 First St.) & jth X.J. & Gold & Bright & 11 \\
\hline 12 & Lorillard, P. Company & Veptune & & ¿th N.J. & Blue & Bright & 12 \\
\hline 13 & McAlpln, D. H. \& Co. & Extra & & 3 XX. & Gold & Bright & $1 ;$ \\
\hline 14 & MIcAlpin, D. H. \& Co. & Hone: Dew & & $3 \mathrm{~d}$ X.Y. & Gold & Bright & 14 \\
\hline 15 & MeAlpin, D. H. \& Co. & Navy & & $3 \mathrm{~d} \times \mathrm{L}$. & Blue & Bright & 15 \\
\hline 16 & McAlpin, D. H. \& Co. & Solace & & $3 d \mathrm{~N} . \mathrm{Y}$. & Gold & Bright & 16 \\
\hline $1 \pi$ & MeAlpln, D. H. \& Co. & Virgin Leaf & & 3d N.Y. & Gold & Bright & 17 \\
\hline 18 & Minler, Mrs. G. B. & Fern Leat & & sI X.Y. & Gold & Brigbt & 16 \\
\hline 19 & Universal Tobacco Co. & Honey Dew & & 2 d XiY. & Gol:] & Bright & 19 \\
\hline 20 & Universal Tobacco Co. & Solace & & - I X.Y. & liold & Bright & 20 \\
\hline
\end{tabular}


1 2-3d OUNCES.

No. Propreter Brand




\section{OUNCE.}

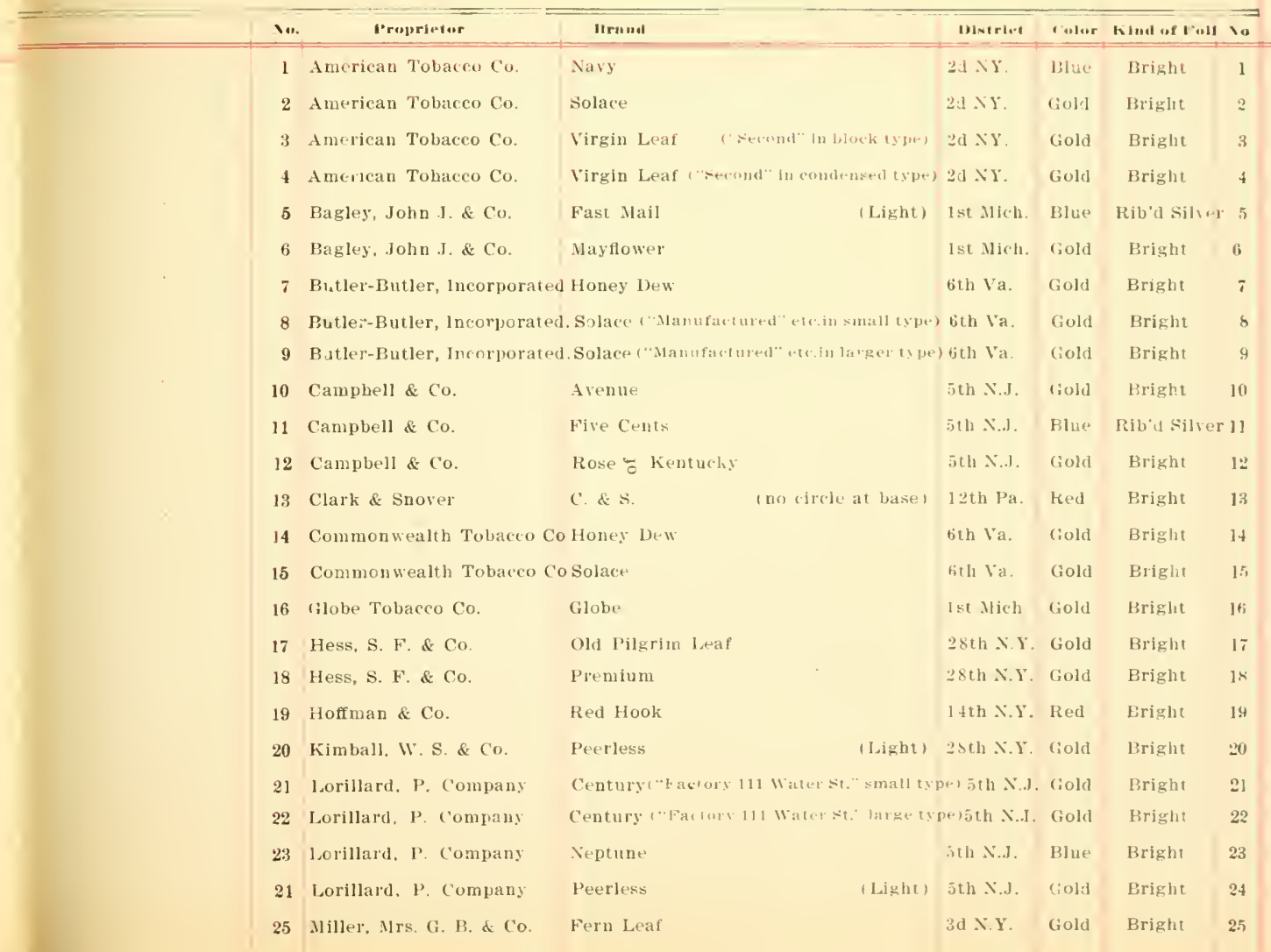


1902 ISSUE.. “Act of April 12, 1902"' (Continued).

FOILS.

1 OUNCE.

Ni.

Proprletur

If rund

binerlet Color lind of Foll vo.

26 Payn's, B. Sons

Golden Strawberry

1 th N.Y. fiold

Bright

1 th N.Y. Gold Bright

27 Shields, Francis

Sweet Cavendish

28 Universal Tobacco Co. Honey Dew

2d N.Y. Gold Bright 28

29 Universal Tobacco Co. Solace

30 Warnick, Brown \& Co. Gold Leat

2d N.Y. Gold Bright 29

21st N.Y. Gold Bright 30 


$$
=
$$

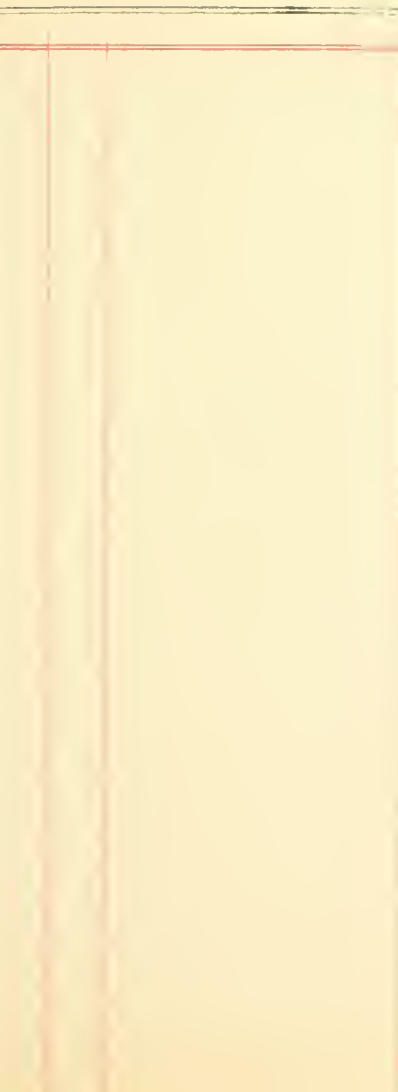


1 2-3d OUNCES.

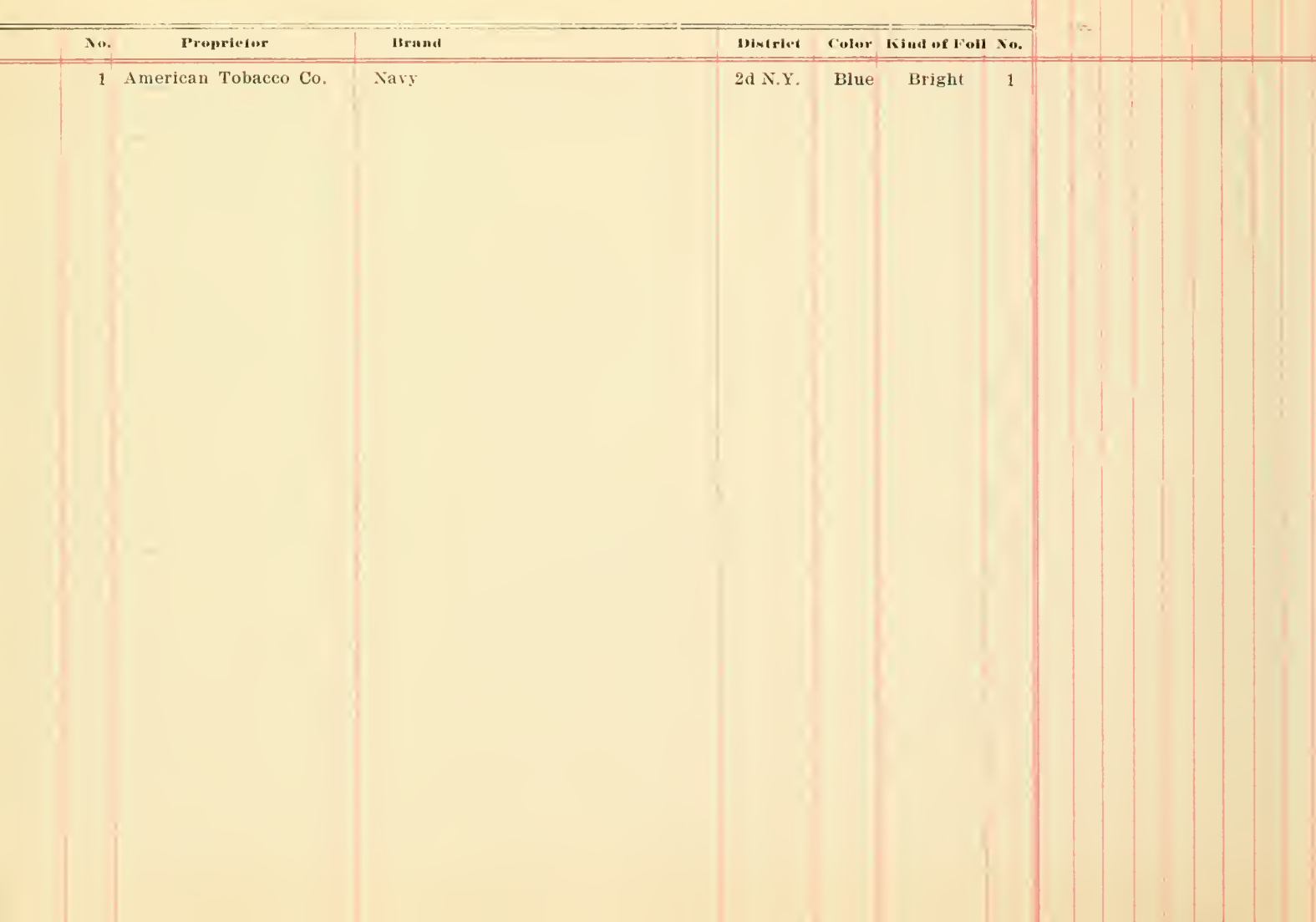





\section{"Class 16c" and "Class 32c."}

\section{1, 2 and 4 OUNCES.}

We have seen cut specimens only of Nos, 2 and 3.

Printed by Continental Bank Note Co.

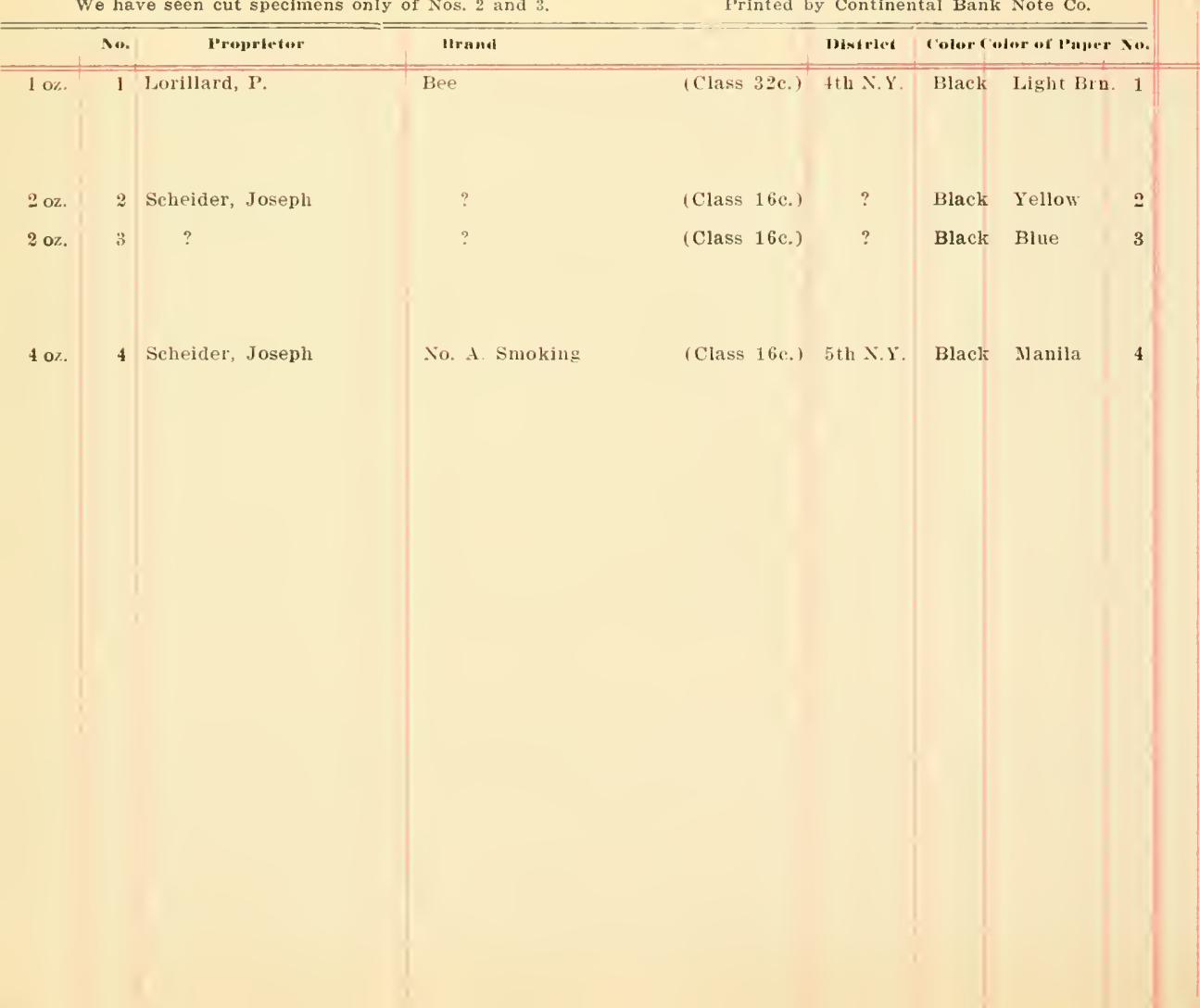




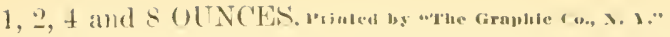

Die A. Head of Liberty in garter. Jle B, Head of Liberty in circle. Dle C, Head of Franklln.

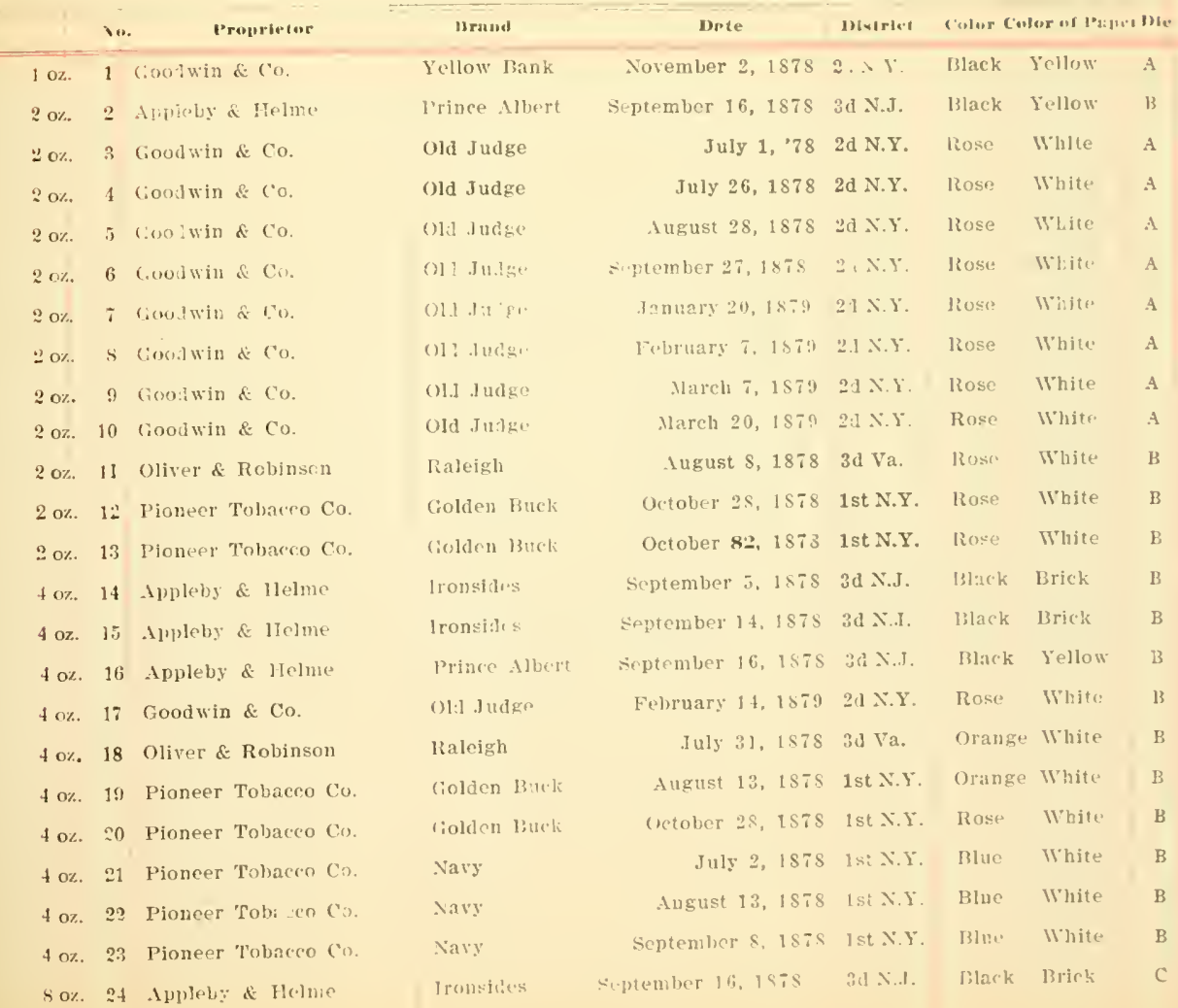


There are 4 varieties of surcharges. * IRed surcharge. o lita sulcharge large. \$ BIue surcharge horizontal. $x$ Blue surcharge vertical. Die $A$, Head of Liberty in garter. Die $B$, Head of Liberty in circle. Dle C, Head of Franklin.

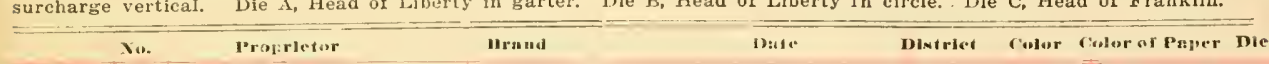

$10 \% \quad 1$ Goodwin \& $\%$

$2 \mathrm{o} \% \quad 2$ Appluby \& Helme

2o\% 3 Goodwin \& Co.

$20 \% 4$ Goodwin \& Co.

$20 \% 5$ Goodwin \& Co.

4 oz. 6 Appleby \& Helme

4 o\%. 7 Appleby \& Heime

4 oz. 8 Appleby \& Helne

4 oz. 9 Goodwin \& Co.

4 oz. 10 Oliver \& Robinson

4 oz. 11 Oliver \& Robinson

4 oz. 12 Oliver \& Robinson

4 oz. 13 Oliver, R. W., Agent

4 oz. 14 Pioneer Tobacco Co.

8 oz. 15 Appleby \& Helme
* Follow bank

* Prince Albert

$\$$ Old Judge

$x$ ()I] Judge

$x$ Old Judge

* Ironsides

* Ironsides

* Prince Albert

$x$ Ola Judge

* Ralejgh

a Kaleigh

* Raleigb

* Raleigh

* Navy

* Ironsides

\begin{tabular}{|c|c|c|c|}
\hline Novenber 2, 1878 & $21 \mathrm{XYY}$. & Black & Yellow \\
\hline etember $16,187 \mathrm{~s}$ & 31] $>. .1$. & Black & Yellow \\
\hline February $i, 1879$ & $2 \mathrm{~d}$ X.Y. & Rose & White \\
\hline February 7,1879 & $2 ₫ N . Y$. & Rose & White \\
\hline Nlarch 7,1879 & Id N.Y. & Rose & White \\
\hline September 5,1878 & $3 \mathrm{~d} \quad \mathrm{~N} . \mathrm{J}$. & Black & Brick \\
\hline September 14,1878 & $3 d \times \mathrm{N}$. & Black & Brick \\
\hline eptember 16,1878 & 3 ก N.J. & Black & Yellow \\
\hline Mareh 20, 1879 & $2 d \mathrm{~N} . \mathrm{Y}$. & Rose & Wbite \\
\hline May 1,1879 & $3 d \mathrm{Va}$. & Orange & White \\
\hline Nov. 16,1879 & $3 \mathrm{~d} \mathrm{Va}$ & Orange & White \\
\hline May 5,1880 & ud Va. & Orange & White \\
\hline March 1,1881 & $3 \mathrm{~d} \mathrm{Va}$ & Orange & White \\
\hline ptember 12,1878 & 1st N.Y. & Bine & White \\
\hline Whombon 18 & 3 & Bla & Yello \\
\hline
\end{tabular}




\section{ISSUE. "Series of 1875 ."}

\section{$10 \mathrm{UN} \mathrm{E}$}

Phose marked $t$ we believe were regularly issued but have never wen a uscd speclmen.

no date. + no date, punched with round hole. \$ dated an l cumplete as finished foil.

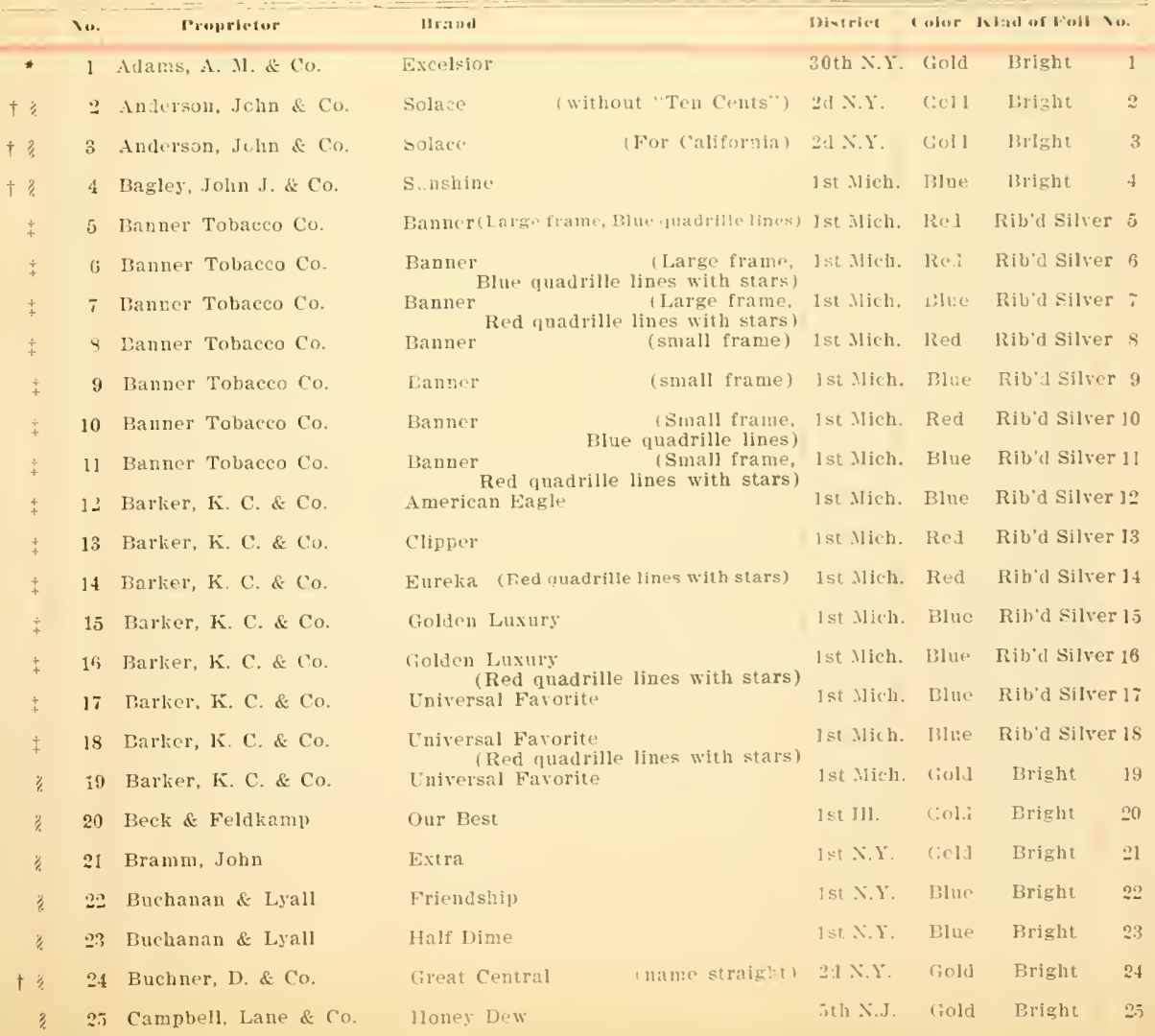


1 ULNC'E.

Thuse marked † we believe were regularly issued hut have nerer seen a used specimen.

* no date. Ino date, punched with round hole. \$ dated and complote as finished foll.

\begin{tabular}{|c|c|c|c|c|c|c|c|c|}
\hline & No. & Proprietor & Brand & & District & Color & Wiad of Foil & so. \\
\hline 8 & $: 6$ & Campbell, Lane \& Co. & Premium & & 5 th N.J. & Gold & Bright & 26 \\
\hline *) & 27 & Campbell, Lane \& Co. & Star & & 5 th N.J. & Gold & Bright & 27 \\
\hline$\xi$ & 28 & Chamberlain,Camplell \& Co & o. Feerless & & 1st Mich. & Gold & Briglit & 24 \\
\hline 3 & $2 !$ & Cliase, lsherwood \& Co. & Mazeppa & & 10 th Ohio & Gold & Bright & 29 \\
\hline$\dagger \hat{3}$ & 30 & Clark \& Snover & Anthracite & & 12 th $\mathrm{Pa}$. & Gold & Bright & 30 \\
\hline 3. & 31 & Cotterill, Fenner \& Co. & Velocipede & & 8d Ohio & Gold & Bright & 31 \\
\hline ? & 32 & Doll \& Co. & Mammoutl Cave & & jth $\mathrm{Ky}$ & Blue & Bright & 32 \\
\hline$\hat{2}$ & 33 & Flint, J, G., Jr. & May Queen & & 1st Wis. & Gold & Bright & 33 \\
\hline ? & $£ 4$ & Frank, W. S. \& Co. & Monareh & & 9th Ky. & Gold & Bright & 34 \\
\hline$?$ & 35 & Gail \& Ax, G. W. & Parade & & $\ddot{d d ~} \mathrm{Vd}$. & Gold & Bright & 35 \\
\hline$+\%$ & 36 & Goodwin \& Co. & Bright Welcome & (Light Welcome) & $2 \mathrm{~d}$ N.Y. & Gold & Bright & 36 \\
\hline$\dagger^{*}$ & 37 & Goodwin \& Co. & Solitaire & & Pd N.Y. & Gold & Bright & 37 \\
\hline$\dagger \%$ & 38 & Goodwin \& Co. & Welcome & (Dark) & 2d N.Y. & Gold & Bright & 38 \\
\hline$t \%$ & $: 39$ & Goodwin \& Co. & Yellow Bank & & $2 \mathrm{~d}$ N.Y. & Gold & Bright & $3 y$ \\
\hline$\%$ & 40 & Glore, J. A. P. \& Bros. & Corn Cracker & & fith $\mathrm{Ky}$. & Gold & Bright & 40 \\
\hline t\% & 41 & Greer's, Alex Sons & Cavenrlish & & 14 th N.Y. & Gold & Bright & 41 \\
\hline$\%$ & 42 & Greer's, Alex Sons & Twist Bud & & $14 \mathrm{th}$ N.Y. & Gold & Bright & 42 \\
\hline 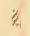 & 43 & Gueker, C. \& G. & Amber & & $\because 8 \mathrm{th}$ N.Y. & Gold & Bright & 43 \\
\hline$\%$ & 44 & Gucker, C. \& G. & Diamond & & $28 \operatorname{th}$ N.Y. & Blue & Bright & 44 \\
\hline
\end{tabular}


1879 PROVISIONAL ISSUE.

FOIL ESSA YS.

sime as 1875 ()mitting "Sieries of $1805 . "$

$10 \mathrm{OUNC}^{\mathrm{T}} \mathrm{C}$.

* no date. no date, punched with round hole. \& dater an l complete as finlshed poil.

No. l'ropreter

? 1 Bagley, John J. \& Co.

?. 2 Bagley, John J. \& Co.

?. 3 Buchner, D. \& Co.

\% 4 Buchner, D. \& Co.

\% 5 Granger, H. \& G. M.

3. 6 Granger, H. \& G. II.

7 Rosenfeld, E.

\% 8 Shields, Francis

* 9 Wallier, JeGraw \& Co.
Bran

Fast Mail

Heart's Content

Inter Ocean

Tidal Wave

Century Nills

Seal of Detrour

Temptation

Sweet Cavendish

Globe
Dintrlet Color filud of Foll Do.

1st Mlich. Blue Bright I

1st Nich. (iold Bright a

(Imp. type 1) 2d X.Y. hed Bright

(1mp. type 1) 2d X.Y. Red Bright 4

(Imp. type 1) 1st Wich. Blue Bright 5

1st Mich. Gold Bright 6

18th Ohio Gold Bright i

$1+$ th XY. Gold Bright 8

1st Mlich. Red Rib'd sllver 9 
1879 ISSUE. "'Series of 1879."

2 OUNCFS.

* no date. : a date, punched with round hole. \$ dated and complete as finished foil.

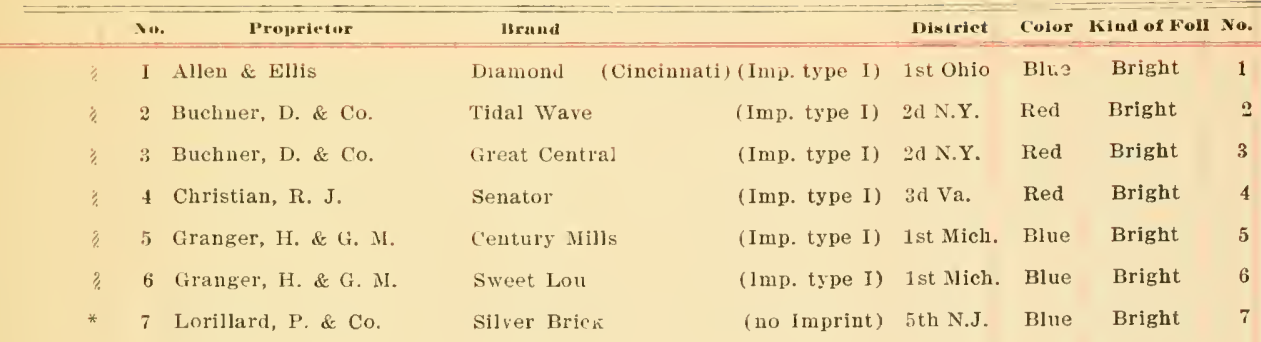


Those narked with a s were listed by E. B. Sitering in 1 sS3 but as we know of no one who has a specimen, we iuclude them here. Nil the others were taken from the recolds of the J. J. Crooke Company by Alfred A. Post, as work done by them but we are inclined to believe that these are simply foil wrappers upon which an adhestve stamp was used

$$
\text { I. }
$$

I'roprleter

Ilen \& Dunning

Bramm, John

Bronson \& Messenger

Buchner, D.

Campbell, Lane \& Co.

Campbell, Lane \& Co.

Douglass, I. S.

Edmonston, Sam'l S.

Franklin Bros.

Grant, H. J.\& Co.

Grant, H. J. \& Co.

fucker, C. N. G.

Hess, F. S.

Hess, F, S.

Hess, F. S.

Hoyt, Thos. \& .J. F. FIagg

Kenny, Thos.

Lang. George

Lichtenberg, G. B.

Lichtenberg, f. B.

Narsh, S.

IIIller. IIrs. G. B.

IIiller, IIrs, G. B.

Payn, Benj.

Robitzek \& Tausig

Scheider, Jos. \& Co.

Warner \& Carater

IVarnick \& Brown

IVarnick \& Brown

Whaler, R \& $T$.

Whitker \& Nash Co.

\section{Is rạual}

Indian $1 \mathrm{Veed}$

Ionitor

Indian Brand

Navy

Honey Dew

Honey Dew

Ruby

Currency

Pleasure

Golden Leaf

Metamora

Diamond

Iontana

Nerada

Premium

Sweet Owen

Veteran

Liberty

dlonument

Iorning Glory

Fine Cut Cavendish

Fine Cut

Queen of the Valley

Strawberry (hewing

Sweet Scented Tobacco

Our Choice

Star

Aromatic Cavendish

Eureka

5

Excelsior
Bixirlite!

Coler biad of leall vo. 


\section{ISSUE. "Class 32 cts."}

$10 \mathrm{OCE}$.

CROOKE RECORDS.

Those marked with a $\$$ were listed by E. B. Sterling in 1583 but as we know of no one who has a suecimen, we Include them here. All the others were taken from the records of the J, J, Crooke Company by Alfred A. Post, as work done by them but we are inclined to believe that these are simply foil wrapprs upon which an adhesive stamp was used

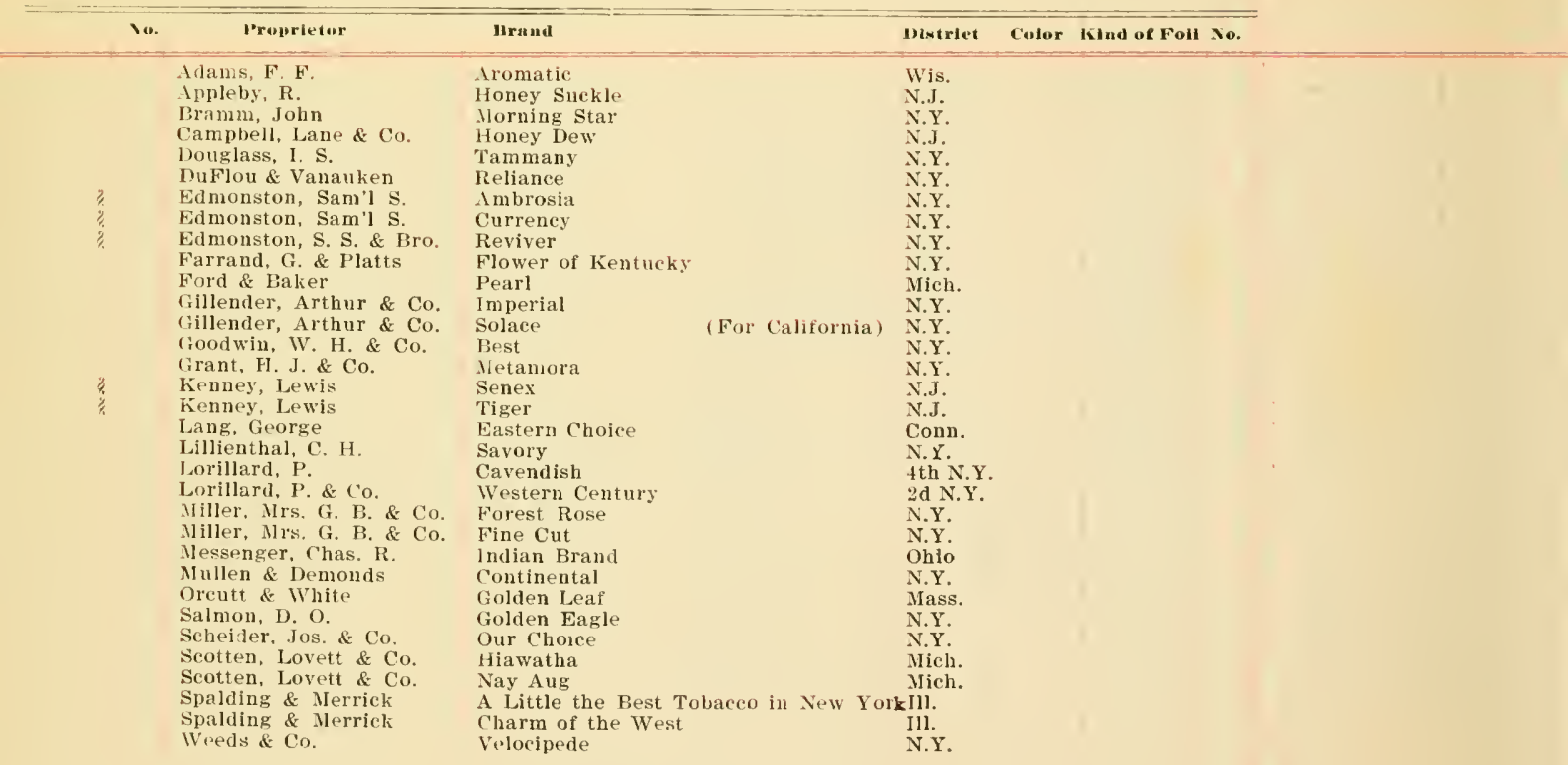




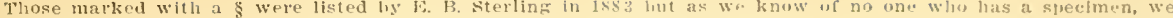

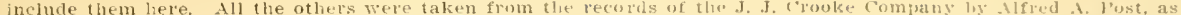
work done by them but we are inclined to believe that these are simsly foll wrappers upron wich an ad iesive stamp was used

$$
\text { 1.. }
$$

1'rupriveter

Itritial

lisisis

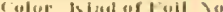

Atams, A. II. \& Co.

tllen, Peynolds \& Co.

Allen, Reynolds \& Co.

Barher, K. C.

Loik \& Wirti

Bramn, John

Lramm, Jehn

Bramm, John

Llow: J. de Co.

Praw11. J. \& Co.

Eromn, d

Frown, J

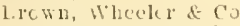

Canabell, Lane de Co.

Caurbeil Lame \& Co.

('amplell, Lane \& Co.

Camplell, Lane \& Cu.

Campbell, Lane \& Co.

Comphell, Lane \& $\mathrm{Co}$

Chasp, Isherwood d Co.

chase, lsliernoort \& ( o.

Clark \& Snoover

('atlin, D.

Erimonston,Sam'l S.\& Bro.

Elmonstou,Sam'l S.\& Bro.

Eimonston,San'l S.\& Bro.

Eimonston,San'l S.\& Bro.

F'inley, Doll \& $\mathrm{Co}$

Finley, Doll \& Co.

Farrand \& Babcock

Franklin \& Bro.

Greer's, Alex Sons

Gucker, C. \& $\mathrm{G}$.

Gucker, C, \& $\mathrm{C}$.

Janna \& $\mathrm{Co}$

IJarris, A. W. \& Co.

Harris, A. W. \& Co.

Harris, A, IV, \& Co.

Harris, A. W. E Co.

Hayten. Thos. S. \& Co.

Hayden, Thos. S. \& Co.

Hoffman \& Co.

Iloyt, Thos, d Co

Kendal, Harrison \& $\mathrm{Co}$

kenny, Thos.

limball. Wm. S.

limball, IVn?. S

kimball, $1 \mathrm{Vm}$. $\mathrm{s}$

Lergat \& Butler

Lescat Hudson \& Butler
Excelsior

Indian Wieed $21: 1$

Universal Favorit,

()ur Best

First Nationa:

, 11 gisiz Linit

Virginia letaf

Libert

Worning (ilo1'y

licse

Goluen Charu

Star

Ilose a kentucliy

lose of kentrely

.r.tis I-tibht

Yoodline

kuse of lingerely

Buckeye

Nazeppa

Anthracite

lolden Throad

Golden Star

Price of the Ralitan

Phoenix

Prile of the I. S

Extra

Iammoth Cave

Flower of lientucliy

Pleasure

Twist Bud

is mer

Diamoud

Premium

Centemial

Golien Etar

Nectar

Jhoenix

Rright Diamond

Golden Fleece

Superior

Hearts Delight

Colden Eagle

senex

traho

Peerless

Peerless Cluewins

iilt Edge
X.Y.

Y.J.

N.J.

Jlich

III.

N.Y

X. $\mathrm{Y}$

11. H. Watts X.Y.

IIeb.

Mich.

ilich.

ilich.

line

Ohio

caldwell. X. J. X..I

caldwell, X. J, ‥

Callwell >े J $\mathrm{x}$.

Patterson, … X. X..

Patterson, $\mathrm{N}$. J. N.J.

Newalk, ‥ J. N. J.

Ohio

Ohio

$\mathrm{Pa}$.

No.

N.Y.

$\mathrm{XY}$.

X.

ㄱ. 1 .

IIy.

lis.

KY.

D. $\mathrm{I}$

X.

N.Y.

$\mathrm{XI}$

N. 1

I. $Y$

I. Y.

X. $Y$

X.

Mass.

Mass.

I. I.

ก.Y.

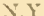

X.J.

$\mathrm{N.Y}$

X.Y.

S.Y.

No.
Mo.

Gold 


\section{$1 \mathrm{OUNCF}$.}

Those marked with a $\$$ were listed by F. B. Sterling in $188: 3$ hut as we know of no one who has a specimen, we include them here. All the others were taken from the recoris of the J. J. "rooke Company by Alfred A. Post, as work done by them but we are inclined to believe that these ale simply foil wappers upon which an adhesive stamp was used

Iraud

Lightoing

..onument

1.ichtenberg, G. B.

lichtenberg. G. B.

1 ichtenlerg, G. B.

Ijlienthal, C. H.

Jilienthal, C. H.

1.orillard, P. \& Co.

lia.tden, Thos. \& Co.

Marsh, S.

Viarsh, S.

Nessenger, Chas. R.

Miller, Mrs. G. B. \& Co.

lliller, Mrs. G. B. \& Co.

Mooney \& Howe

MulJen \& Demond

Mliller, Egbert

Nicks \& Hoffman

Payn, Benj.

Payn, Benj.

Rice \& Carmichael

Rice \& Seger

Rice \& Seger

Rice, W. H.

Richardson, 11. \& Son

Rosenfeld, w.

Rosenfeld, H. S. \& Co.

Scottell, Lovett \& Co.

Senour, Simrall \& Noonan

Shields \& Son

schriber, J.

Schriber, J.

Spalding \& Merrick

Thomas \& Pilkinton

Thomas \& Pilkinton

Warner \& Crater

Warnick \& Brown

Watts, J. H.

Watts, L. H.

Watts, L. H.

Wratts, L. H.

Watts, L. H.

Withers, J. E. \& Co.

Witker, Nash $\propto$ Co.

Wolf, J. P. \& Co.

Whalen, R. \& $T$.

Wheeler, John A. \& Co. violet

Savory

Young America

Violet

Highlan'l Gem

Highlander

-..ighlanciel.

Granger

Grape

Prize Leaf

Social

Boquet

Bonuet

Superior

Collen Strawbery

Strawherry Chewing

Sweet Consoler

Honey Dew

$\rightarrow$ weet Consolar.

Honey Dew

Union Chewing

Gclden Leaf

Golden Leaf

Nay Aug

standard Kentuck.

weet Cavendish

A A Chewing

Prairie Flower

Charm of the IVest

indispensable

rirginia

Sunbeam

Aromatic

Tobaceo Leaf

fiolden

Virginia leaf

Fine cut

specie

uid Necessity

Excelsior

Dayton Belle

Tip Top

(iolden Charn
Dixtriet

fober tolual of tioji In. 


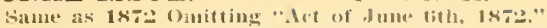

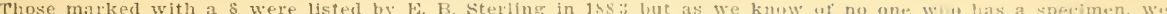

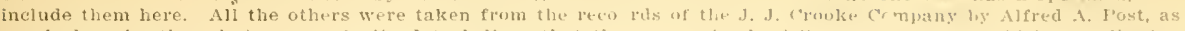
work done by them but we are inelined to believe that these it s simply ioll wrappess upn whlch an adhesite stamp was usea

\begin{tabular}{|c|c|c|c|}
\hline No. & Drobriecor & 3rand & iviri \\
\hline & 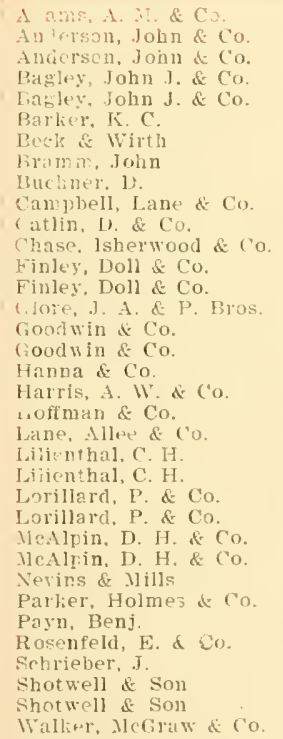 & 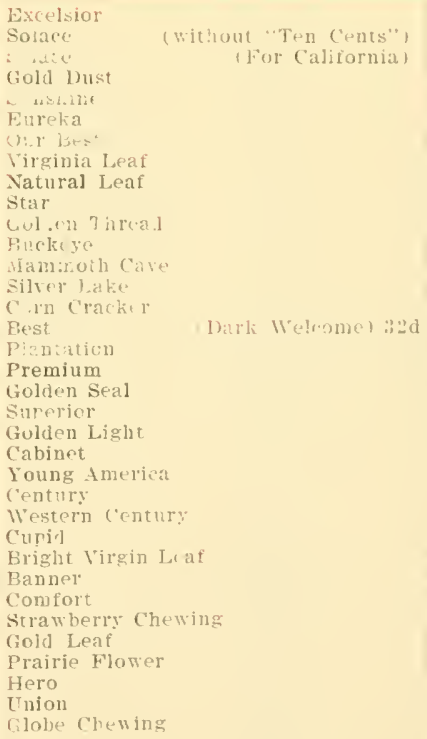 & 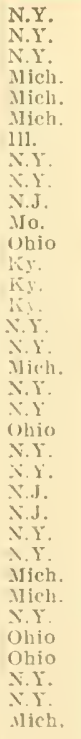 \\
\hline
\end{tabular}




\section{ISEUE. “'Series of 1875.'}

Those marked with a s were listed by E. B. Sterling in $18 \mathrm{~s}:$ wit as we know of no one who has a specimen, we include them here. All the others were taken from the recolds of the .J. J. Crouke Company by Alfred A. Post, as work done by them but we are inclined to believe that these are simply foil wrappers upon which an adhesive stamp was used

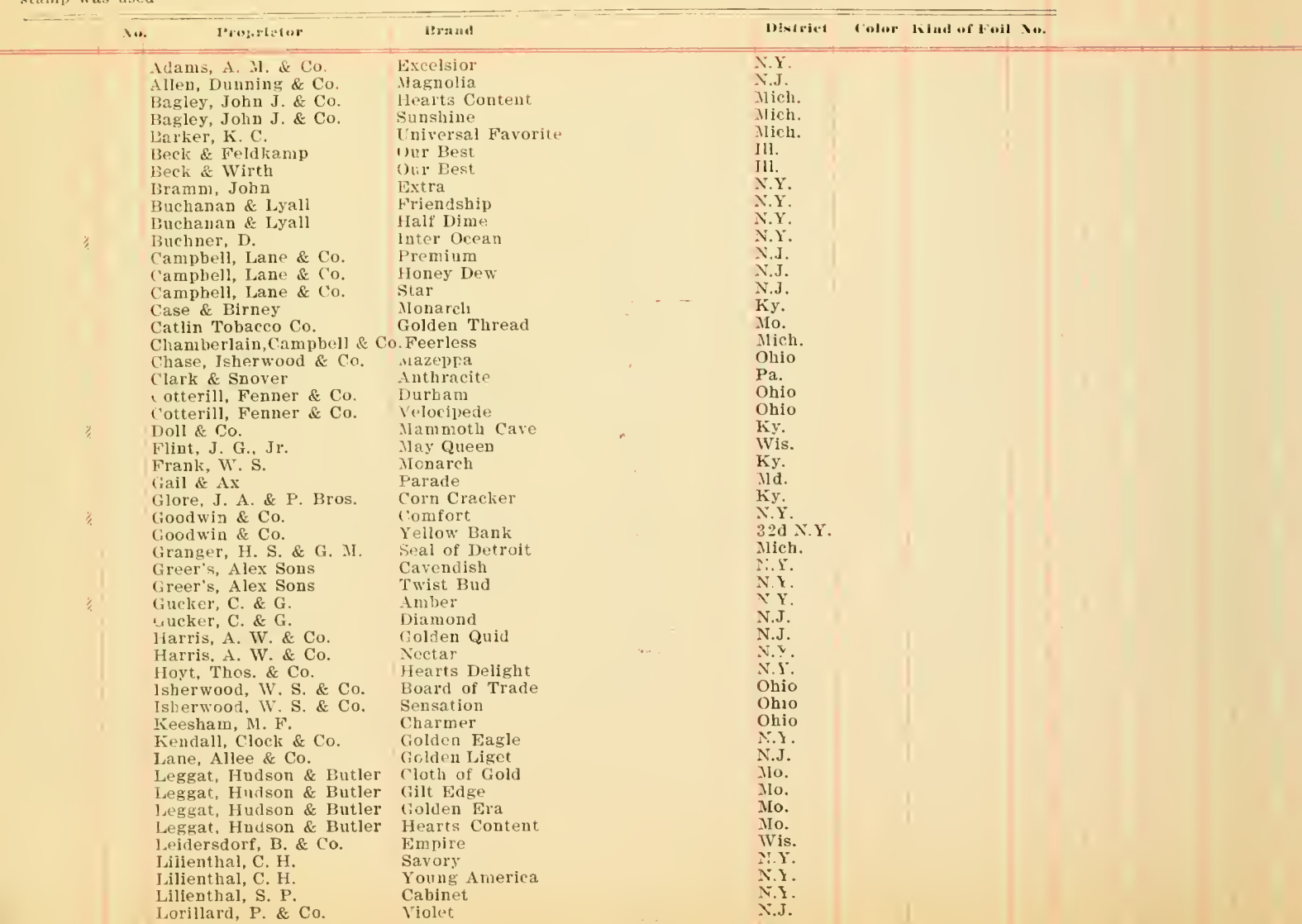


Those makked with a $\$$ were ltsted by E. B. Sterling in 1883 but as we know of no one who has a speclmen, we

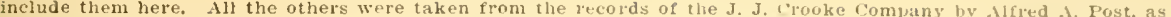
work done by them but we are inclined to helleve that these are simply foll wraplers upon which an adheslve statin was used

\4.

Hrand

Meliride, Jas. G. \& Co.

Miarsh, s.

Miller, Mrs. G. B. \& Co.

IIull

Morten Bros.

Power \& Stewart

Richarison. Ml. \& Co.

Rosenfeld \& Co.

Scliriber, J.

Schriber, J

Shields, Francis

Varnick \& Brow

Weighell, M. B. W.

Weighell, M. B. Y

Whaler, R. \& T.

Williams, $T$. $f$ Co.

llhjtier, Halsted e Co.
Rose Bud

superb

Novity.

Boquet

S.immit

Board of Tracle

Enion ('htwing

Gold Leaf

A A Chewing

Prairie Flow r

sweet Cavendisl

Iromatic

Cavelier

Liglitning

Tip Top

Let T's Have Peare

Excelsios biatries

Coior lilnal of Full Do.

Mich.

․?

x.

$\mathrm{Ky}$.

Ohjo

Ohio

Ohjo

Ohio

Ohio

A.

N.Y

()hio

Ohio

‥

Ia.

Obio 


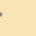



$$
1
$$ 
$3 \underset{\text { SMITHSONAAN INSTITUTION LiBRAMIES }}{9088}$ 